ALEA, Lat. Am. J. Probab. Math. Stat. 18, 907-943 (2021)

DOI: 10.30757/ALEA.v18-33

\title{
Spatial averages for the parabolic Anderson model driven by rough noise
}

\author{
David Nualart, Xiaoming Song and Guangqu Zheng \\ University of Kansas, Department of Mathematics \\ 1460 Jayhawk Blvd, Lawrence, KS 66045, USA \\ E-mail address: nualart@ku.edu \\ $U R L:$ https://nualart.ku.edu/ \\ Drexel University, Department of Mathematics \\ 3141 Chestnut Street, Philadelphia, PA 19104, USA \\ E-mail address: xs73@drexel.edu \\ URL: https://www.math.drexel.edu/ song/ \\ University of Kansas, Department of Mathematics \\ 1460 Jayhawk Blvd, Lawrence, KS 66045, USA \\ E-mail address: zhengguangqu@gmail.com; corresponding author \\ URL: https://gzheng1990.com/
}

\begin{abstract}
In this paper, we study spatial averages for the parabolic Anderson model in the Skorohod sense driven by rough Gaussian noise, which is colored in space and time. We include the case of a fractional noise with Hurst parameters $H_{0}$ in time and $H_{1}$ in space, satisfying $H_{0} \in(1 / 2,1), H_{1} \in(0,1 / 2)$ and $H_{0}+H_{1}>3 / 4$. Our main result is a functional central limit theorem for the spatial averages. As an important ingredient of our analysis, we present a Feynman-Kac formula that is new for these values of the Hurst parameters.
\end{abstract}

\section{Introduction}

We consider the Parabolic Anderson Model $(\mathrm{PAM})$ on $\mathbb{R}_{+} \times \mathbb{R}$

$$
\left\{\begin{array}{l}
\frac{\partial u}{\partial t}=\frac{1}{2} \Delta u+u \diamond \dot{W}, \\
u(0, \cdot) \equiv 1,
\end{array}\right.
$$

Received by the editors October 12th, 2020; accepted February 11th, 2021.

2010 Mathematics Subject Classification. 60H15, 60H07, 60G15, 60F05.

Key words and phrases. Parabolic Anderson model, fractional rough noise, Malliavin calculus, central limit theorem, Wiener chaos expansion, Feynman-Kac formula, fourth moment theorems.

David Nualart is supported by the NSF Grant DMS 1811181. 
where $\dot{W}(t, x)$ is a generalized centered Gaussian random field with covariance

$$
\mathbb{E}[\dot{W}(t, x) \dot{W}(s, y)]=\gamma_{0}(t-s) \gamma(x-y) .
$$

The product in (1.1) is the Wick product and the mild solution is defined in the Skorohod sense. We assume that the covariance (1.2) satisfies one of the following two (overlapping) sets of conditions:

(H1) $\gamma_{0}: \mathbb{R} \rightarrow[0, \infty]$ is a nonnegative-definite locally integrable function and $\gamma$ is a tempered distribution, whose Fourier transform $\mu$ admits a density $\varphi$ that satisfies the following modified Dalang's condition:

$$
\int_{\mathbb{R}} \frac{\varphi(x)^{2}}{1+x^{2}} d x<+\infty
$$

We also assume that $\varphi$ is continuous at zero with $\varphi(0)=0$ and the following concavity condition is satisfied: $\exists \kappa_{0} \in(0, \infty)$ such that

$$
\varphi(x+y) \leq \kappa_{0}[\varphi(x)+\varphi(y)] \text { for every } x, y \in \mathbb{R} .
$$

(H2) $\gamma_{0}(t)=|t|^{2 H_{0}-2}$ for some $H_{0} \in(1 / 2,1)$ and $\varphi(z)=|z|^{1-2 H_{1}}$ for some $H_{1} \in(0,1 / 2)$ such that ${ }^{1} H_{0}+H_{1}>3 / 4$.

The existence of a unique mild solution under condition (H1) has been established in Huang et al. (2017a,b). Notice, that unlike many of the works on the stochastic heat equation with spatial colored noise, in (H1) the correlation function $\gamma$ does not need to be a measure. Previously the random field solution theory for the stochastic heat equation driven by a Gaussian noise white in time and rough in space, was restricted to the range $H_{1} \in(1 / 4,1 / 2)$ (see, for instance, Balan et al., 2015; Hu et al., 2017, 2018). Condition $H_{0}+H_{1}>3 / 4$ in case (H2) breaks the barrier $1 / 4$, which is required under $(\mathbf{H 1})$ if $\varphi(x)=|x|^{1-2 H_{1}}$, and it has been observed in Song et al. (2020) that the random field solution exists for the PAM when $H_{0} \in(1 / 2,1), H_{1} \in(0,1 / 2)$ and $H_{0}+H_{1}>3 / 4$.

We are interested in deriving a functional central limit theorem (CLT) for spatial averages of the form

$$
A_{t}(R):=\int_{-R}^{R}[u(t, x)-1] d x
$$

as $R \rightarrow \infty$, where $t \in[0, \infty)$. Using the chaos expansion of the solution (see (2.3)) and a chaotic CLT (see Theorem 1.4)), we prove the following main result.

Theorem 1.1. Let $A_{t}(R)$ be defined as in (1.3) and suppose that one of the assumptions (H1) or (H2) holds. Then, as $R \rightarrow \infty$,

$\left\{\frac{1}{\sqrt{R}} A_{t}(R)\right\}_{t \in \mathbb{R}_{+}}$converges in law to a centered Gaussian process $\mathcal{G}$ on $C\left(\mathbb{R}_{+} ; \mathbb{R}\right)$, where for any $t_{1}, t_{2} \in \mathbb{R}_{+}$,

$$
\mathbb{E}\left[\mathcal{G}_{t_{1}} \mathcal{G}_{t_{2}}\right]=2 \int_{\mathbb{R}} \mathbb{E}\left[\mathfrak{g}\left(\mathcal{I}_{t_{1}, t_{2}}^{1,2}(z)\right)\right] d z
$$

with $\mathfrak{g}(z)=e^{z}-z-1$ and $\mathcal{I}_{t_{1}, t_{2}}^{1,2}(z)$ being the random variable defined in Proposition 1.3.

\footnotetext{
${ }^{1}$ In the fractional case we actually have $\varphi(z)=c\left(H_{1}\right)|z|^{1-2 H_{1}}$, with $c\left(H_{1}\right)=\frac{\sin \left(\pi H_{1}\right)}{2 \pi} \Gamma\left(2 H_{1}+\right.$ 1 ), but to simplify the presentation we take $c\left(H_{1}\right)=1$.
} 
Since the work Huang et al. (2020a) of Huang, Nualart and Viitasaari, there have been a series of papers devoted to central limit theorems for spatial averages of stochastic partial differential equations. The authors of Huang et al. (2020a) consider the nonlinear stochastic heat equation driven by space-time white noise on $\mathbb{R}_{+} \times \mathbb{R}$ and they are able to provide a functional central limit theorem for $A_{t}(R)$. Their methodology begins with rewriting $A_{t}(R)=\delta\left(V_{t, R}\right)$ as a Skorohod integral and then appeal to the recent Malliavin-Stein approach (Nourdin and Peccati, 2012) to obtain a quantitative CLT for $A_{t}(R)$ in total variation distance. The convergence of finite-dimensional distributions can be proved using the multivariate MalliavinStein bound and the tightness is established using Kolmogorov's criterion. Other key tools used in Huang et al. (2020a) are Clark-Ocone formula, Burkholder's inequality and they essentially rely on the assumption that the underlying Gaussian noise is white in time so as to render us a martingale structure. Soon later, the authors of Huang et al. (2020b) consider the same equation with spatial dimension $d \geq 1$; while imposing that the Gaussian noise is white in time and it has a spatial covariance given by the Riesz kernel, they establish a functional CLT and a quantitative CLT for spatial averages. We also refer interested readers to several other investigations on stochastic heat equations in Chen et al. (2019, 2020); Gu and Li (2020); Khoshnevisan et al. (2020); Pu (2020) and on stochastic wave equations in Nualart and Zheng (2020b); Delgado-Vences et al. (2020); Nualart and Zheng (2020c); these papers more or less follow the path paved by the work Huang et al. (2020a), although the nature of the problems and the techniques differ.

In the above references, the underlying Gaussian noise is always white in time, which is crucial to apply stochastic calculus techniques. These techniques are not available in our framework because the noise is colored in time, which forces to restrict our study to linear equations, where we can use Wiener chaos expansions and Feynman-Kac formulas. In Nualart and Zheng (2020a), that is the closest work to the present paper, Nualart and Zheng consider the PAM (1.1) on $\mathbb{R}_{+} \times \mathbb{R}^{d}$ with the correlation kernels $\gamma_{0}$ and $\gamma$ satisfying the following conditions:

(i) $\gamma_{0}: \mathbb{R} \rightarrow[0, \infty]$ is nonnegative-definite and locally integrable.

(ii) $\gamma$ is a positive finite measure, expressed as the Fourier transform of some nonnegative tempered measure $\mu$ that satisfies Dalang's condition (Dalang, 1999; Hu et al., 2015) $\int_{\mathbb{R}^{d}} \frac{\mu(d \xi)}{1+\|\xi\|^{2}}<\infty$, where $\|\cdot\|$ denotes the Euclidean norm.

In Nualart and Zheng (2020a), the Gaussian fluctuation is established for

$$
\int_{\{\|x\| \leq R\}}[u(t, x)-1] d x
$$

for each $t>0$ and, under the extra integrability condition on $\gamma_{0}$

$$
\int_{0}^{t_{0}} \int_{0}^{t_{0}} \gamma_{0}(s-t) s^{-\alpha} t^{-\alpha} d s d t<\infty, \quad \text { for some } \alpha \in(0,1 / 2), t_{0}>0,
$$

the functional CLT holds as well; see Nualart and Zheng (2020a, Theorems 1.6, 1.9) for more precise statements. Note also that in our Theorem 1.1, we do not need to assume condition (1.4) used to guarantee the tightness.

Observe that unlike in the papers Huang et al. (2020b); Delgado-Vences et al. (2020), the variance order of $A_{t}(R)$ is $R$, which does not depend on the parameters of the covariance, for example, the Hurst index $H_{1}$ in the setting (H2). This is due 
to our assumption $\varphi(0)=0$ in both settings of (H1) and (H2), that forces the negligibility of the first chaotic component of $A_{t}(R)$ in the limit, while the other chaoses contribute to the order $R$. This situation is completely different from the case $H_{1}>1 / 2$, where a nonchaotic behavior occurs.

In what follows, we briefly sketch the main steps of the proof of Theorem 1.1. The first step in proving Theorem 1.1 is to show the order of the limiting variance and more generally, to establish the limit covariance structure.

Proposition 1.2. Assume hypothesis $(\mathbf{H 1})$ or $(\mathbf{H 2})$. For $t_{1}, t_{2} \in \mathbb{R}_{+}$,

$$
\frac{1}{2 R} \operatorname{Cov}\left(A_{t_{1}}(R), A_{t_{2}}(R)\right) \stackrel{R \rightarrow \infty}{\longrightarrow} \int_{\mathbb{R}} d z \mathbb{E}\left[e^{\mathcal{I}_{t_{1}, t_{2}}^{1,2}(z)}-\mathcal{I}_{t_{1}, t_{2}}^{1,2}(z)-1\right],
$$

where $\mathcal{I}_{t_{1}, t_{2}}^{1,2}(z)$ is defined in Proposition 1.3. Moreover if $\Pi_{p}(\bullet)$ denotes the orthogonal projection onto the pth Wiener chaos associated to $W$ (see Section 2 for more details), we have for $p=1$,

$$
\lim _{R \rightarrow \infty} \frac{1}{2 R} \operatorname{Cov}\left(\Pi_{1} A_{t_{1}}(R), \Pi_{1} A_{t_{2}}(R)\right)=0,
$$

and for each $p \geq 2$,

$$
\lim _{R \rightarrow \infty} \frac{1}{2 R} \operatorname{Cov}\left(\Pi_{p} A_{t_{1}}(R), \Pi_{p} A_{t_{2}}(R)\right)=\frac{1}{p !} \int_{\mathbb{R}} d z \mathbb{E}\left[\left(\mathcal{I}_{t_{1}, t_{2}}^{1,2}(z)\right)^{p}\right] .
$$

An important ingredient to prove Proposition 1.2 is the following Feynman-Kac formula for the moments of the solution. In the case (H1), this formula is essentially a reformulation of Corollary 4.4 in Huang et al. (2017a) and the result for (H2) is new, so we provide in Section 6 a unified proof for both cases.

Proposition 1.3. In both cases $(\mathbf{H 1})$ and $\mathbf{( H 2 )}$, we have for any $\left(s_{i}, t_{i}, x_{i}, y_{i}\right) \in$ $(0, \infty)^{2} \times \mathbb{R}^{2}, i=1, \ldots, k(k \geq 2)$

$$
\mathbb{E}\left[\prod_{i=1}^{k} u\left(t_{i}, x_{i}\right)\right]=\mathbb{E}\left[\exp \left(\sum_{1 \leq i<j \leq k} \mathcal{I}_{t_{i}, t_{j}}^{i, j}\left(x_{i}-x_{j}\right)\right)\right],
$$

where

$$
\mathcal{I}_{t, s}^{i, j}(z):=\int_{0}^{t} \int_{0}^{s} d r d v \gamma_{0}(r-v) \int_{\mathbb{R}} d \xi \varphi(\xi) e^{-\mathbf{i}\left(B_{t-r}^{i}-B_{s-v}^{j}+z\right) \xi}
$$

is understood as the $L^{p}(\Omega)$-limit (for any $p \geq 1$ ) of

$$
\int_{0}^{t} \int_{0}^{s} d r d v \gamma_{0}(r-v) \int_{\mathbb{R}} d \xi e^{-\varepsilon \xi^{2}} \varphi(\xi) e^{-\mathbf{i}\left(B_{t-r}^{i}-B_{s-v}^{j}+z\right) \xi}=: \mathcal{I}_{t, s, \varepsilon}^{i, j}(z),
$$

with $B^{1}, \ldots, B^{k}$ being i.i.d. standard Brownian motions on $\mathbb{R}$. Moreover, for each $i<j$ and for any $\lambda>0$,

$$
\sup \left\{\mathbb{E}\left[\exp \left(\lambda\left|\mathcal{I}_{t, s, \varepsilon}^{i, j}(z)\right|\right)\right]: \varepsilon>0, z \in \mathbb{R}\right\}<\infty
$$

Once Proposition 1.2 is proved, we apply the multivariate chaotic central limit theorem to establish the convergence in law of finite-dimensional distributions (f.d.d.). This chaotic CLT is a consequence of the well-known fourth moment theorems (Nourdin and Peccati, 2012; Nualart and Peccati, 2005; Peccati and Tudor, 2005). 
Theorem 1.4 (Multivariate chaotic CLT). Fix an integer $n \geq 1$ and consider a family $\left\{A_{R}: R>0\right\}$ of centered random vectors in $\mathbb{R}^{n}$ such that each component of $A_{R}=\left(A_{R, 1}, \ldots, A_{R, n}\right)$ belongs to $L^{2}(\Omega, \sigma\{W\}, \mathbb{P})$ and has the following chaos expansion

$$
A_{R, j}=\sum_{q=1}^{\infty} I_{q}^{W}\left(g_{q, j, R}\right) \quad \text { with } g_{q, j, R} \in \mathfrak{H}^{\odot q}
$$

where $I_{q}^{W}$ denotes the qth-multiple stochastic integral with respect to $W$ (see Section 2). Suppose the following conditions (a)-(d) hold:

(a) $\forall i, j \in\{1, \ldots, n\}$ and $\forall q \geq 1, \mathbb{E}\left[I_{q}^{W}\left(g_{q, j, R}\right) I_{q}^{W}\left(g_{q, i, R}\right)\right] \stackrel{R \rightarrow+\infty}{\longrightarrow} \sigma_{i, j, q}$.

(b) $\forall i \in\{1, \ldots, n\}, \sum_{q=1}^{\infty} \sigma_{i, i, q}<\infty$.

(c) For any $1 \leq r \leq q-1,\left\|g_{q, i, R} \otimes_{r} g_{q, i, R}\right\|_{\mathfrak{H}^{\otimes(2 q-2 r)}} \stackrel{R \rightarrow+\infty}{\longrightarrow} 0$.

(d) $\forall i \in\{1, \ldots, n\}, \lim _{N \rightarrow+\infty} \sup _{R>0} \sum_{q=N+1}^{\infty} \mathbb{E}\left[I_{q}^{W}\left(g_{q, i, R}\right)^{2}\right]=0$.

Then $A_{R}$ converges in law to $N(0, \Sigma)$ as $R \rightarrow+\infty$, where $\Sigma=\left(\sigma_{i, j}\right)_{i, j=1}^{n}$ is given by $\sigma_{i, j}=\sum_{q=1}^{\infty} \sigma_{i, j, q}$.

We refer to Campese et al. (2020); Nourdin and Peccati (2012); Hu and Nualart (2005) for more details on this result and to Section 2.2 for the definition of the Hilbert space $\mathfrak{H}$ and the $r$-contraction $\otimes_{r}$ in our setting. As the last step in the proof of Theorem 1.1, we will establish the tightness by using Kolmogorov's criterion. The key tool is the hypercontractivity property of the Ornstein-Uhlenbeck generator, see (2.1).

Remark 1.5. Theorem 1.1 and Proposition 1.3 also hold under Hypothesis (H1) if $\gamma_{0}=\delta_{0}$, that means, if the noise is white in time. The proofs are similar and we omit the details. In this framework, the random variables $\mathcal{I}_{t, s, \varepsilon}^{i, j}(z)$ defined in Proposition 1.3 would have the expression

$$
\int_{0}^{t \wedge s} d r \int_{\mathbb{R}} d \xi e^{-\varepsilon \xi^{2}} \varphi(\xi) e^{-\mathbf{i}\left(B_{t-r}^{i}-B_{s-r}^{j}+z\right) \xi}
$$

The rest of this article is organized as follows: Section 2 is devoted to preliminary knowledge that is required for later proofs, and we prove Proposition 1.2 in Section 3; we show the f.d.d. convergence and tightness respectively in Section 4 and Section 5; the last section contains the proof of Feynman-Kac formula.

\section{Preliminaries}

We first introduce some handy notation here.

2.1. Notation. For $r \in \mathbb{N}$ and $x_{\boldsymbol{r}}=\left(x_{1}, \ldots, x_{r}\right)$, we write $d x_{\boldsymbol{r}}=d x_{1} \ldots d x_{r}$, $\mu\left(d \boldsymbol{x}_{\boldsymbol{r}}\right)=\mu\left(d x_{1}\right) \ldots \mu\left(d x_{r}\right)$ and $\tau\left(\boldsymbol{x}_{\boldsymbol{r}}\right)=x_{1}+\cdots+x_{r}$. For integers $1 \leq r<p$, we write $\left(\xi_{1}, \ldots, \xi_{p}\right)=\boldsymbol{\xi}_{\boldsymbol{p}}=\left(\boldsymbol{\xi}_{\boldsymbol{r}}, \boldsymbol{\eta}_{\boldsymbol{p}-\boldsymbol{r}}\right)$ with $\boldsymbol{\xi}_{\boldsymbol{r}}=\left(\xi_{1}, \ldots, \xi_{r}\right)$ and $\boldsymbol{\eta}_{\boldsymbol{p}-\boldsymbol{r}}=\left(\xi_{r+1}, \ldots, \xi_{p}\right)$.

For any $p \in \mathbb{N}, \mathfrak{S}_{p}$ denotes the set of permutations of $\{1,2, \ldots, p\}$, and for any $\boldsymbol{s}_{\boldsymbol{p}}=\left(s_{1}, \ldots, s_{p}\right)$ we write $\boldsymbol{s}_{\boldsymbol{p}}^{\boldsymbol{\sigma}}=\left(s_{\sigma(1)}, \ldots, s_{\sigma(p)}\right)$. 
For any interval $I$, we use $|I|$ to denote its length.

For any $t>0$ and $m \in \mathbb{N}$, put $\Delta_{m}(t):=\left\{\boldsymbol{r}_{m} \in \mathbb{R}_{+}^{m}: t>r_{1}>\cdots>r_{m}>0\right\}$ and $\operatorname{SIM}_{m}(t):=\left\{\boldsymbol{w}_{\boldsymbol{m}} \in \mathbb{R}_{+}^{m}: w_{1}+\cdots+w_{m} \leq t\right\}$.

For any $p>0$ and any random variable $X \in L^{p}(\Omega)$, we write $\|X\|_{p}=$ $\left(\mathbb{E}\left[|X|^{p}\right]\right)^{1 / p}$.

2.2. Wiener chaos expansion. For $\phi, \psi \in C_{c}^{\infty}\left(\mathbb{R}_{+} \times \mathbb{R}\right)$, we define

$$
\langle\phi, \psi\rangle_{\mathfrak{H}}=\int_{\mathbb{R}_{+}^{2}} d s d t \gamma_{0}(s-t) \int_{\mathbb{R}} d \xi \varphi(\xi) \mathcal{F} \phi(s, \xi) \mathcal{F} \psi(t,-\xi)
$$

where $\mathcal{F} \phi(s, \xi):=\int_{\mathbb{R}} e^{-\mathbf{i} x \xi} \phi(s, x) d x$ is the Fourier transform with respect to the spatial variable only. Due to our assumptions ((H1) or (H2)), the above functional $(\phi, \psi) \longmapsto\langle\phi, \psi\rangle_{\mathfrak{H}}$ defines an inner product, under which $C_{c}^{\infty}\left(\mathbb{R}_{+} \times \mathbb{R}\right)$ can be extended to a Hilbert space, denoted by $\mathfrak{H}$.

We can view the noise $W$ as an isonormal Gaussian process over $\mathfrak{H}$, that is, $\{W(h): h \in \mathfrak{H}\}$ is a centered Gaussian family with

$$
\mathbb{E}[W(\phi) W(\psi)]=\langle\phi, \psi\rangle_{\mathfrak{H}},
$$

for any $\phi, \psi \in \mathfrak{H}$. For any $n \in \mathbb{N}$, we denote by $\mathfrak{H}^{\otimes n}$ the $n$th tensor product of $\mathfrak{H}$ and by $\mathfrak{H}^{\odot n}$ the symmetric subspace of $\mathfrak{H}^{\otimes n}$.

It is a well-known fact that $L^{2}(\Omega, \sigma\{W\}, \mathbb{P})$ can be decomposed into an infinite orthogonal sum:

$$
L^{2}(\Omega, \sigma\{W\}, \mathbb{P})=\bigoplus_{p=0}^{\infty} \mathbb{C}_{p}
$$

where $\mathbb{C}_{p}$ is called the $p$ th Wiener chaos and it is the $L^{2}(\Omega)$-completion of the set

$$
\left\{H_{p}(W(\psi)):\|\psi\|_{\mathfrak{H}}=1\right\},
$$

with $H_{p}(x)=(-1)^{p} e^{x^{2} / 2} \frac{d^{p}}{d x^{p}} e^{-x^{2} / 2}$ denoting the $p$ th Hermite polynomial. For any integer $p \geq 1$, the multiple integral $I_{p}^{W}$ of order $p$ is a bounded linear operator from $\mathfrak{H}^{\otimes p}$ onto $\mathbb{C}_{p}$ uniquely characterized by the following conditions:

(i) Given any orthogonal unit vectors $e_{1}, \ldots, e_{k} \in \mathfrak{H}(k \geq 2)$ and any nonnegative integers $n_{1}, \ldots, n_{k}$ such that $n_{1}+\cdots+n_{k}=p$, it holds that

$$
I_{p}^{W}\left(e_{1}^{\otimes n_{1}} \otimes e_{2}^{\otimes n_{2}} \otimes \cdots \otimes e_{k}^{\otimes n_{k}}\right)=\prod_{i=1}^{k} H_{n_{i}}\left(W\left(e_{i}\right)\right) .
$$

(ii) For any $f \in \mathfrak{H}^{\otimes p}, I_{p}^{W}(f)=I_{p}^{W}(\widetilde{f})$, where $\widetilde{f} \in \mathfrak{H}^{\odot p}$ denotes the symmetrization of $f$.

For $p=0, \mathbb{C}_{0}=\mathbb{R}$ and $I_{0}$ is the identity. The following isometry property holds for any $f \in \mathfrak{H}^{\otimes p}$ and $g \in \mathfrak{H}^{\otimes q}$ (see Nourdin and Peccati, 2012; Nualart, 2006 for more details):

$$
\mathbb{E}\left[I_{p}^{W}(f) I_{q}^{W}(g)\right]=p !\langle\widetilde{f}, \widetilde{g}\rangle_{\mathfrak{H} \otimes p} \mathbf{1}_{\{p=q\}} .
$$

Another important property of Wiener chaos is the following consequence of hypercontractivity (see e.g. Nourdin and Peccati, 2012, Corollary 2.8.14): If $F \in \mathbb{C}_{p}$ for $p \geq 1$, then for any $k \geq 2$,

$$
\|F\|_{k} \leq(k-1)^{p / 2}\|F\|_{2}
$$


Let us introduce the contractions appearing in condition (c) of Theorem 1.4. For integers $p, q \geq 1$, the $r$-contraction $f \otimes_{r} g$ for $f \in \mathfrak{H}^{\otimes p}, g \in \mathfrak{H}^{\otimes q}$ and $1 \leq r \leq p \wedge q$ is the element of $\mathfrak{H}^{\otimes(p+q-2 r)}$ defined by

$$
f \otimes_{r} g=\sum_{i_{1}, \ldots, i_{r}=1}^{\infty}\left\langle f, e_{i_{1}} \otimes \cdots \otimes e_{i_{r}}\right\rangle_{\mathfrak{H}^{\otimes r}}\left\langle g, e_{i_{1}} \otimes \cdots \otimes e_{i_{r}}\right\rangle_{\mathfrak{H}^{\otimes r}}
$$

where $\left\{e_{i}, i \geq 1\right\}$ is a complete orthonormal system in $\mathfrak{H}$. In the particular case where $f$ and $g$ are locally integrable functions, the contraction $f \otimes_{r} g$ has the following expression

$$
\begin{aligned}
&\left(f \otimes_{r} g\right)\left(\boldsymbol{t}_{\boldsymbol{p}-\boldsymbol{r}}, \boldsymbol{\xi}_{\boldsymbol{p}-\boldsymbol{r}} ; \boldsymbol{s}_{\boldsymbol{q}-\boldsymbol{r}}, \boldsymbol{\eta}_{\boldsymbol{q}-\boldsymbol{r}}\right)=\int_{\mathbb{R}_{+}^{2 r} \times \mathbb{R}^{r}}\left(\mathcal{F}_{r} f\right)\left(\boldsymbol{t}_{\boldsymbol{p}-\boldsymbol{r}}, \boldsymbol{\xi}_{\boldsymbol{p}-\boldsymbol{r}}, \boldsymbol{a}_{\boldsymbol{r}}, \boldsymbol{\zeta}_{\boldsymbol{r}}\right) \\
& \times\left(\mathcal{F}_{r} g\right)\left(\boldsymbol{s}_{\boldsymbol{q}-\boldsymbol{r}}, \boldsymbol{\eta}_{\boldsymbol{q}-\boldsymbol{r}}, \boldsymbol{b}_{\boldsymbol{r}},-\boldsymbol{\zeta}_{\boldsymbol{r}}\right) \prod_{j=1}^{r} \gamma_{0}\left(a_{j}-b_{j}\right) \varphi\left(\zeta_{j}\right) d a_{j} d b_{j} d \zeta_{j},
\end{aligned}
$$

where $\mathcal{F}_{r}$ denotes the Fourier transform with respect to the $r$ space variables. We refer readers to the appendix of Nourdin and Peccati (2012) for more explanations on the contractions.

2.3. Malliavin calculus. We will denote by $D$ the derivative operator in the sense of Malliavin calculus. That is, if $F$ is a smooth and cylindrical random variable of the form

$$
F=f\left(W\left(h_{1}\right), \ldots, W\left(h_{n}\right)\right),
$$

with $h_{i} \in \mathfrak{H}$ and $f \in C_{c}^{\infty}\left(\mathbb{R}^{n}\right)$, then $D F$ is the $\mathfrak{H}$-valued random variable defined by

$$
D F=\sum_{j=1}^{n} \frac{\partial f}{\partial x_{j}}\left(W\left(h_{1}\right), \ldots, W\left(h_{n}\right)\right) h_{j}
$$

The Sobolev space $\mathbb{D}^{1,2}$ is the closure of the space of smooth and cylindrical random variables under the norm

$$
\|D F\|_{1,2}=\sqrt{\mathbb{E}\left[F^{2}\right]+\mathbb{E}\left[\|D F\|_{\mathfrak{H}}^{2}\right]}
$$

We denote by $\delta$ the adjoint of the derivative operator given by the duality formula

$$
\mathbb{E}(\delta(u) F)=\mathbb{E}\left(\langle D F, u\rangle_{\mathfrak{H}}\right)
$$

for any $F \in \mathbb{D}^{1,2}$ and any $u \in L^{2}(\Omega ; \mathfrak{H})$ in the domain of $\delta$. The operator $\delta$ is also called the Skorohod integral because in the case of the Brownian motion, it coincides with an extension of the Itô integral introduced by Skorohod. We refer to Nualart (2006) for a detailed account of the Malliavin calculus with respect to a Gaussian process.

If $F \in \mathbb{D}^{1,2}$ and $h$ is a deterministic element of $\mathfrak{H}$, then $F h$ is Skorohod integrable and, by definition, the Wick product $F \diamond W(h)$ equals the Skorohod integral of $F h$, that is,

$$
\delta(F h)=F \diamond W(h)
$$


2.4. Mild solution. Now we define

$$
\mathcal{F}_{t}=\sigma\left\{W(\phi): \phi \in C_{c}^{\infty}\left(\mathbb{R}_{+} \times \mathbb{R}\right) \text { has support contained in }[0, t] \times \mathbb{R}\right\} \vee \mathcal{N},
$$

where $\mathcal{N}$ denotes the collection of null sets. This gives us a filtration $\mathbb{F}:=\left\{\mathcal{F}_{t}: t \geq\right.$ $0\}$.

For each $t>0$ and $x \in \mathbb{R}, G(t, x)=(2 \pi t)^{-1 / 2} e^{-x^{2} /(2 t)}$ denotes the fundamental solution of the heat equation.

Definition 2.1. An $\mathbb{F}$-adapted random field $u=\{u(t, x): t \geq 0, x \in \mathbb{R}\}$ is a mild solution to (1.1) if for all $(t, x) \in \mathbb{R}_{+} \times \mathbb{R}$, the process $\left\{G(t-s, x-y) u(s, y) \mathbf{1}_{[0, t]}(s)\right.$ : $\left.(s, y) \in \mathbb{R}_{+} \times \mathbb{R}\right\}$ is Skorohod integrable and following integral equation holds

$$
u(t, x)=1+\int_{0}^{t} \int_{\mathbb{R}} G(t-s, x-y) u(s, y) W(d s, d y),
$$

where the stochastic integral is understood in the Skorohod sense.

From the results of Huang et al. (2017b,a); Nualart and Zheng (2020a), under hypotheses (H1) or (H2), there exists a unique mild solution to equation (1.1), which has the following Wiener chaos expansion

$$
u(t, x)=1+\sum_{n=1}^{\infty} I_{n}^{W}\left(f_{t, x, n}\right)
$$

where $f_{t, x, n} \in \mathfrak{H}^{\odot n}$ is given by

$$
f_{t, x, n}\left(\boldsymbol{s}_{\boldsymbol{n}}, \boldsymbol{y}_{\boldsymbol{n}}\right)=\frac{1}{n !} G\left(t-s_{\sigma(1)}, x-y_{\sigma(1)}\right) \prod_{i=1}^{n-1} G\left(s_{\sigma(i)}-s_{\sigma(i+1)}, y_{\sigma(i)}-y_{\sigma(i+1)}\right)
$$

with $\sigma \in \mathfrak{S}_{n}$ such that $t>s_{\sigma(1)}>\ldots>s_{\sigma(n)}>0$; we refer readers to Hu et al. (2015); Huang et al. (2017a); Song et al. (2020) for the rigorous derivation of (2.3).

Then the core object in this paper $A_{t}(R)$ has the following Wiener chaos expansion

$$
A_{t}(R)=\sum_{p=1}^{\infty} I_{p}^{W}\left(\int_{-R}^{R} f_{t, x, p} d x\right)
$$

as a consequence of stochastic Fubini.

In the end of this section, let us state a useful embedding result that is a consequence of the Hardy-Littlewood inequality.

2.5. Embedding of $L^{1 / H_{0}}\left(\mathbb{R}_{+}^{n}\right)$ into $\mathcal{H}^{\otimes n}$. Let $\mathcal{H}$ be the Hilbert space associated to the fractional Brownian motion with Hurst parameter $H_{0} \in(1 / 2,1)$. That means, $\mathcal{H}$ is the closure of $C_{c}^{\infty}\left(\mathbb{R}_{+}\right)$under the seminorm

$$
\langle f, g\rangle_{\mathcal{H}}=\int_{\mathbb{R}_{+}} f(s) g(t)|s-t|^{2 H_{0}-2} d s d t .
$$

We have the continuous embedding $L^{1 / H_{0}}\left(\mathbb{R}_{+}\right) \hookrightarrow \mathcal{H}$. More precisely, for any $f, g: \mathbb{R}_{+} \rightarrow \mathbb{R}$ with $\|f\|_{L^{1 / H_{0}\left(\mathbb{R}_{+}\right)}}<\infty$ and $\|g\|_{L^{1 / H_{0}\left(\mathbb{R}_{+}\right)}}<\infty$, we have

$$
\left|\langle f, g\rangle_{\mathcal{H}}\right| \leq C_{H_{0}}\|f\|_{L^{1 / H}\left(\mathbb{R}_{+}\right)}\|g\|_{L^{1 / H}\left(\mathbb{R}_{+}\right)},
$$


where the constant $C_{H_{0}}$ only depends on $H_{0}$; see, for instance, Mémin et al. (2001). Then, by iteration, one can easily show that given two functions $f, g \in L^{1 / H_{0}}\left(\mathbb{R}_{+}^{n}\right)$, then

$$
\begin{aligned}
\left|\langle f, g\rangle_{\mathcal{H} \otimes n}\right| & =\left|\int_{\mathbb{R}_{+}^{2 n}} d s_{1} \cdots d s_{n} d t_{1} \cdots d t_{n} f\left(s_{1}, \ldots, s_{n}\right) g\left(t_{1}, \ldots, t_{n}\right) \prod_{j=1}^{n}\right| s_{j}-\left.t_{j}\right|^{2 H_{0}-2} \mid \\
& \leq C_{H_{0}}^{n}\|f\|_{L^{1 / H_{0}\left(\mathbb{R}_{+}^{n}\right)}}\|g\|_{L^{1 / H_{0}}\left(\mathbb{R}_{+}^{n}\right)} .
\end{aligned}
$$

\section{Limiting covariance structure}

For any nonnegative measurable function $\Theta$ and for any integer $p \geq 1$, we define the following two quantities:

$$
\begin{aligned}
& K_{1, p}(\Theta, t)=\int_{[0, t]^{p}} d \boldsymbol{s}_{\boldsymbol{p}} \int_{\mathbb{R}^{p}} \mu\left(d \boldsymbol{\xi}_{\boldsymbol{p}}\right) \Theta\left(\tau\left(\boldsymbol{\xi}_{\boldsymbol{p}}\right)\right) \exp \left[-\frac{1}{2} \operatorname{Var} \sum_{j=1}^{p} B_{s_{j}} \xi_{j}\right] \\
& K_{2, p}(\Theta, t)=\int_{[0, t]^{p}} d \boldsymbol{s}_{\boldsymbol{p}}\left(\int_{\mathbb{R}^{p}} \mu\left(d \boldsymbol{\xi}_{\boldsymbol{p}}\right) \Theta\left(\tau\left(\boldsymbol{\xi}_{\boldsymbol{p}}\right)\right) \exp \left[-\operatorname{Var} \sum_{j=1}^{p} B_{s_{j}} \xi_{j}\right]\right)^{\frac{1}{2 H_{0}}},
\end{aligned}
$$

where, here and along the paper, $B$ denotes a standard real-valued Brownian motion.

Notice that integrating on the simplex $\Delta_{p}(t)$ and making the change of variables $\eta_{j}=\xi_{1}+\cdots+\xi_{j}$ and $s_{j-1}-s_{j}=w_{j}, j=1, \ldots, p$, with the convention $\eta_{0}=0$ and $s_{0}=0$, we obtain

$$
\begin{aligned}
& K_{1, p}(\Theta, t)=p ! \int_{\operatorname{SIM}_{p}(t)} d \boldsymbol{w}_{\boldsymbol{p}} \int_{\mathbb{R}^{p}} d \boldsymbol{\eta}_{\boldsymbol{p}} \Theta\left(\eta_{p}\right) e^{-\frac{1}{2} \sum_{k=1}^{p} w_{k} \eta_{k}^{2}} \prod_{j=1}^{p} \varphi\left(\eta_{j}-\eta_{j-1}\right) \\
& K_{2, p}(\Theta, t)=p ! \int_{\operatorname{SIM}_{p}(t)} d \boldsymbol{w}_{\boldsymbol{p}}\left(\int_{\mathbb{R}^{p}} d \boldsymbol{\eta}_{\boldsymbol{p}} \Theta\left(\eta_{p}\right) e^{-\sum_{k=1}^{p} w_{k} \eta_{k}^{2}} \prod_{j=1}^{p} \varphi\left(\eta_{j}-\eta_{j-1}\right)\right)^{\frac{1}{2 H_{0}}}
\end{aligned}
$$

The next technical lemma will play a fundamental role along the paper.

Lemma 3.1. Under hypothesis (H1), we have, for any $N>0$,

$$
K_{1, p}(\Theta, t) \leq p ! t\left(\int_{\mathbb{R}} \Theta(x)(1+\varphi(x)) d x\right) \frac{1}{2}\left(8 \kappa_{0} C_{N}\right)^{p-1} \exp \left(\frac{t D_{N}}{2 C_{N}}\right),
$$

where the constants $C_{N}$ and $D_{N}$, given by

$$
C_{N}:=\int_{\{|\eta| \geq N\}} \frac{1+\varphi(\eta)+\varphi(\eta)^{2}}{\eta^{2}} d \eta \quad \text { and } \quad D_{N}:=\int_{\{|\eta| \leq N\}}\left(1+\varphi(\eta)+\varphi(\eta)^{2}\right) d \eta
$$

are finite for each $N>0$ due to the modified Dalang's condition ${ }^{2}$ (D). Moreover

$$
K_{1, p}(1, t) \leq \frac{p !}{2 \kappa_{0}}\left(8 \kappa_{0} C_{N}\right)^{p} \exp \left(\frac{t D_{N}}{2 C_{N}}\right) .
$$

\footnotetext{
${ }^{2}$ Condition (D) implies $\int_{\mathbb{R}} \frac{\varphi(x)+\varphi(x)^{2}}{1+x^{2}} d x<\infty$.
} 
Under hypotheses (H2), we have

$$
K_{2, p}(\Theta, t) \leq p !\left(\int_{\mathbb{R}} \Theta(x)\left(1+|x|^{1-2 H_{1}}\right) d x\right) \frac{C_{1}^{p}\left(t^{p \mathfrak{h}+\frac{1-H_{1}}{2 H_{0}}} \vee t^{p \mathfrak{h}+\frac{1}{4 H_{0}}}\right)}{\Gamma\left(p \mathfrak{h}+\frac{1-H_{1}}{2 H_{0}}\right) \wedge \Gamma\left(p \mathfrak{h}+\frac{1}{4 H_{0}}\right)},
$$

where $\mathfrak{h}=\frac{2 H_{0}+H_{1}-1}{2 H_{0}}>\frac{1}{4} ;$ moreover,

$$
K_{2, p}(1, t) \leq p ! C_{2}^{p} \frac{t^{p \mathfrak{h}}}{\Gamma(p \mathfrak{h}+1)},
$$

where $C_{1}$ and $C_{2}$ are constants depending only on $H_{0}$ and $H_{1}$.

Remark 3.2. Notice that inequalities (3.7) and (3.9) cannot be obtained from (3.5) and (3.8), respectively, by simply putting $\Theta=1$.

Proof of Lemma 3.1: Let us first show the inequality (3.7) under hypothesis (H1). We will make use of the expression (3.3), where $\Theta=1$. Using the concavity condition $(\mathrm{C})$ and the fact that $\varphi(-x)=\varphi(x)$, we can write

$$
\prod_{k=1}^{p} \varphi\left(\eta_{k}-\eta_{k-1}\right) \leq \kappa_{0}^{p-1} \sum_{\beta \in \mathcal{A}_{p}} \prod_{k=1}^{p} \varphi\left(\eta_{k}\right)^{\beta_{k}}
$$

where $\mathcal{A}_{p}$ is a collection of the indices $\boldsymbol{\beta}=\left(\beta_{1}, \ldots, \beta_{p}\right) \in\{0,1,2\}^{p}$ satisfying

$$
x_{1} \prod_{j=2}^{p}\left(x_{j}+x_{j-1}\right)=\sum_{\boldsymbol{\beta} \in \mathcal{A}_{p}} \prod_{k=1}^{p} x_{k}^{\beta_{k}} .
$$

It is easy to see that the cardinality of $\mathcal{A}_{p}$ is $2^{p-1}$. Plugging the inequality (3.10) into (3.3) yields

$$
K_{1, p}(1, t) \leq p ! \kappa_{0}^{p-1} \sum_{\boldsymbol{\beta} \in \mathcal{A}_{p}} \int_{\operatorname{SIM}_{p}(t)} d \boldsymbol{w}_{\boldsymbol{p}} \int_{\mathbb{R}^{p}} d \boldsymbol{\eta}_{\boldsymbol{p}} e^{-\frac{1}{2} \sum_{k=1}^{p} w_{k} \eta_{k}^{2}} \prod_{j=1}^{p} \varphi\left(\eta_{j}\right)^{\beta_{j}} .
$$

Following the same arguments as in the proof of Lemma 3.3 in Hu et al. (2015), we have

$$
K_{1, p}(1, t) \leq p !\left(2 \kappa_{0}\right)^{p-1} \sum_{k=0}^{p}\left(\begin{array}{l}
p \\
k
\end{array}\right) \frac{t^{k}}{k !}\left(D_{N}\right)^{k}\left(2 C_{N}\right)^{p-k},
$$

where the quantities $C_{N}$ and $D_{N}$ are defined in (3.6). The sum in the right-hand side of (3.11) can be estimated as follows:

$$
\sum_{k=0}^{p}\left(\begin{array}{l}
p \\
k
\end{array}\right) \frac{t^{k}}{k !}\left(D_{N}\right)^{k}\left(2 C_{N}\right)^{p-k}=\left(2 C_{N}\right)^{p} \sum_{k=0}^{p}\left(\begin{array}{l}
p \\
k
\end{array}\right) \frac{\left(t D_{N}\right)^{k}}{\left(2 C_{N}\right)^{k} k !} \leq\left(4 C_{N}\right)^{p} e^{\frac{t D_{N}}{2 C_{N}}} .
$$

Substituting (3.12) into (3.11) yields (3.7).

To show (3.5), using the expression (3.3), the estimates

$$
\varphi\left(\eta_{p}-\eta_{p-1}\right) \leq \kappa_{0}\left[\varphi\left(\eta_{p}\right)+\varphi\left(\eta_{p-1}\right)\right] \quad \text { and } \quad \exp \left(-\frac{1}{2} w_{p} \eta_{p}^{2}\right) \leq 1,
$$

we write

$$
\begin{aligned}
& K_{1, p}(\Theta, t) \\
& =p t \kappa_{0}\left(\int_{\mathbb{R}} \Theta\left(\eta_{p}\right) \varphi\left(\eta_{p}\right) d \eta_{p}\right) K_{1, p-1}(1, t)+p t \kappa_{0}\left(\int_{\mathbb{R}} \Theta\left(\eta_{p}\right) d \eta_{p}\right) \widetilde{K}_{1, p-1}(1, t),
\end{aligned}
$$


where

$$
\begin{gathered}
\widetilde{K}_{1, p-1}(1, t)=(p-1) ! \int_{\operatorname{SIM}_{p-1}(t)} d \boldsymbol{w}_{\boldsymbol{p - 1}} \int_{\mathbb{R}^{p-1}} d \boldsymbol{\eta}_{\boldsymbol{p}-\mathbf{1}} e^{-\frac{1}{2} \sum_{k=1}^{p-1} w_{k} \eta_{k}^{2}} \\
\times\left(\prod_{j=1}^{p-1} \varphi\left(\eta_{j}-\eta_{j-1}\right)\right) \varphi\left(\eta_{p-1}\right) .
\end{gathered}
$$

It is easy to show that the estimate (3.7) still holds if we replace $K_{1, p}(1, t)$ by $\widetilde{K}_{1, p}(1, t)$. Therefore, substituting the estimate

$$
\max \left(K_{1, p-1}(1, t), \widetilde{K}_{1, p-1}(1, t)\right) \leq \frac{(p-1) !}{2 \kappa_{0}}\left(8 \kappa_{0} C_{N}\right)^{p-1} \exp \left(\frac{t D_{N}}{2 C_{N}}\right)
$$

into (3.13), we obtain (3.5).

Now let us prove (3.8) under hypothesis (H2). We observe that, using the expression (3.4), we can write

$$
\begin{aligned}
& K_{2, p}(\Theta, t) \\
= & p ! \int_{\operatorname{SIM}_{p}(t)} d \boldsymbol{w}_{\boldsymbol{p}}\left(\int_{\mathbb{R}} d \eta_{p} \Theta\left(\eta_{p}\right) \int_{\mathbb{R}^{p-1}} d \boldsymbol{\eta}_{\boldsymbol{p}-\mathbf{1}} e^{-\sum_{k=1}^{p} w_{k} \eta_{k}^{2}} \prod_{j=1}^{p} \varphi\left(\eta_{j}-\eta_{j-1}\right)\right)^{\frac{1}{2 H_{0}}} \\
\leq & p ! \int_{\operatorname{SIM}_{p}(t)} d \boldsymbol{w}_{\boldsymbol{p}}\left(\sum_{\boldsymbol{\beta} \in \mathcal{A}_{p}} \int_{\mathbb{R}} d \eta_{p} \Theta\left(\eta_{p}\right) \int_{\mathbb{R}^{p-1}} d \boldsymbol{\eta}_{\boldsymbol{p}-\mathbf{1}} e^{-\sum_{k=1}^{p} w_{k} \eta_{k}^{2}} \prod_{j=1}^{p}\left|\eta_{j}\right|^{\beta_{j}\left(1-2 H_{1}\right)}\right)^{\frac{1}{2 H_{0}}} \\
\leq & p ! \sum_{\boldsymbol{\beta} \in \mathcal{A}_{p}} \int_{\operatorname{SIM}_{p}(t)} d \boldsymbol{w}_{\boldsymbol{p}}\left(\int_{\mathbb{R}} d \eta_{p} \Theta\left(\eta_{p}\right) \int_{\mathbb{R}^{p-1}} d \boldsymbol{\eta}_{\boldsymbol{p}-\mathbf{1}} e^{-\sum_{k=1}^{p} w_{k} \eta_{k}^{2}} \prod_{j=1}^{p}\left|\eta_{j}\right|^{\beta_{j}\left(1-2 H_{1}\right)}\right)^{\frac{1}{2 H_{0}}} .
\end{aligned}
$$

For $\beta \in \mathcal{A}_{p}$, we have $\beta_{p} \in\{0,1\}$. As a consequence,

$$
\begin{aligned}
K_{2, p}(\Theta, t) & \leq C_{\Theta} t p ! \sum_{\boldsymbol{\beta} \in \mathcal{A}_{p}} \int_{\operatorname{SIM}_{p-1}(t)} d \boldsymbol{w}_{\boldsymbol{p}-\mathbf{1}} \prod_{j=1}^{p-1}\left(\int_{\mathbb{R}} d \eta_{j} e^{-w_{j} \eta_{j}^{2}}\left|\eta_{j}\right|^{\beta_{j}\left(1-2 H_{1}\right)}\right)^{\frac{1}{2 H_{0}}} \\
& =C_{\Theta} t p ! \sum_{\boldsymbol{\beta} \in \mathcal{A}_{p}} \prod_{j=1}^{p} K\left(\beta_{j}\left(1-2 H_{1}\right)\right)^{\frac{1}{2 H_{0}}} \int_{\operatorname{SIM}_{p-1}(t)} d \boldsymbol{w}_{\boldsymbol{p}-1} \prod_{j=1}^{p-1} w_{j}^{-\frac{1+\beta_{j}\left(1-2 H_{1}\right)}{4 H_{0}}},
\end{aligned}
$$

where $C_{\Theta}=\int_{\mathbb{R}} \Theta(x)\left(1+|x|^{1-2 H_{1}}\right) d x$ and we use the notation

$$
K(\theta):=\int_{\mathbb{R}} e^{-x^{2}} x^{\theta} d x, \theta \geq 0 .
$$

It is clear that by our assumptions on $\left(H_{0}, H_{1}\right)$, that the quantities $\alpha_{j}:=$ $-\frac{1+\beta_{j}\left(1-2 H_{1}\right)}{4 H_{0}}$ belong to the interval $(-1,0)$, so that

$$
\begin{aligned}
\int_{\operatorname{SIM}_{p-1}(t)} d \boldsymbol{w}_{\boldsymbol{p}-\mathbf{1}} \prod_{j=1}^{p-1} w_{j}^{-\frac{1+\beta_{j}\left(1-2 H_{1}\right)}{4 H_{0}}} & =\int_{\operatorname{SIM}_{p-1}(t)} d \boldsymbol{w}_{\boldsymbol{p}-\mathbf{1}} \prod_{j=1}^{p-1} w_{j}^{\alpha_{j}} \\
& =\frac{t^{p-1+\alpha_{1}+\cdots+\alpha_{p-1}}}{\Gamma\left(p+\alpha_{1}+\cdots+\alpha_{p-1}\right)} \prod_{i=1}^{p-1} \Gamma\left(1+\alpha_{i}\right)
\end{aligned}
$$


see also Lemma 6.2 in Song et al. (2020). As $\beta_{1}+\cdots+\beta_{p-1} \in\{p-1, p\}$, then

$$
\sum_{j=1}^{p-1} \alpha_{j} \in\left\{-(p-1) \frac{1-H_{1}}{2 H_{0}}, \frac{1}{4 H_{0}}-p \frac{1-H_{1}}{2 H_{0}}\right\}
$$

and $p+\alpha_{1}+\ldots+\alpha_{p-1} \in\left\{p \mathfrak{h}+\frac{1-H_{1}}{2 H_{0}}, p \mathfrak{h}+\frac{1}{4 H_{0}}\right\}$ with $\mathfrak{h}=\frac{2 H_{0}+H_{1}-1}{2 H_{0}}>\frac{1}{4}$. Thus,

$$
\Gamma\left(p+\alpha_{1}+\cdots+\alpha_{p-1}\right) \geq \Gamma\left(p \mathfrak{h}+\frac{1-H_{1}}{2 H_{0}}\right) \wedge \Gamma\left(p \mathfrak{h}+\frac{1}{4 H_{0}}\right) .
$$

Then, inequality (3.8) follows from the previous computations. The inequality (3.9) has been proved in Song et al. (2020, Remark 3.3).

Remark 3.3. Suppose that we choose $\Theta(x)=G(a, x)$ in Lemma 3.1, for some $a \in(0,1)$. Then, if $\delta>0$ is such that $\varphi$ is bounded in $[-\delta, \delta]$, we obtain, under hypothesis (H1) or (H2),

$$
\int_{\mathbb{R}} d x G(a, x) \varphi(x) d x \leq \sup _{x \in[-\delta, \delta]} \varphi(x)+\sqrt{a} \int_{\{|x| \geq \delta\}} d x \frac{\varphi(x)}{x^{2}},
$$

which implies

$$
\mathcal{C}_{0}=\sup _{a \in(0,1)} \int_{\mathbb{R}} G(a, x)(1+\varphi(x)) d x<\infty .
$$

Next, we will give a useful estimate that will be applied in many places. For any $R>0$, we define

$$
\ell_{R}(\xi)=\frac{\sin ^{2}(R \xi)}{\pi R \xi^{2}}
$$

and note that

$$
\int_{[-R, R]^{2}} d x d y e^{-\mathbf{i}(x-y) \xi}=\frac{4 \sin ^{2}(R \xi)}{\xi^{2}}=4 \pi R \ell_{R}(\xi) .
$$

By Nualart and Zheng (2020a, Lemma 2.1), $\left\{\ell_{R}\right\}_{R>0}$ defines an approximation to the identity (as $R \rightarrow \infty)$.

Lemma 3.4. Assume (H1) or (H2). For any $\varepsilon>0$, there exists a constant $C(\varepsilon)$ such that

$$
\int_{\mathbb{R}} \ell_{R}(\xi) \varphi(\xi) d \xi \leq \varepsilon+\frac{C(\varepsilon)}{R} .
$$

Proof: In both cases (H1) and (H2), the function $\varphi$ is continuous at 0 . Thus, for any $\varepsilon>0$, there exists some $\delta>0$ such that $\varphi(x)<\varepsilon$ for $|x|<\delta$. Then,

$$
\int_{\{|\xi|<\delta\}} \ell_{R}(\xi) \varphi(\xi) d \xi \leq \varepsilon \int_{\mathbb{R}} \ell_{R}(\xi) d \xi=\varepsilon,
$$

and

$$
\int_{\{|\xi| \geq \delta\}} \ell_{R}(\xi) \varphi(\xi) d \xi \leq\left\{\begin{array}{l}
\frac{1}{\pi R} \int_{\{|\xi| \geq \delta\}} \frac{\varphi(\xi)}{\xi^{2}} d \xi \leq \frac{C(\varepsilon)}{R} \text { in case (H1), } \\
\frac{1}{\pi R} \int_{\{|\xi| \geq \delta\}}|\xi|^{-1-2 H_{1}} d \xi \leq \frac{C(\varepsilon)}{R} \text { in case (H2). }
\end{array}\right.
$$

In this way, we just proved (3.16). 
Remark 3.5. If we choose $\Theta(x)=\ell_{R}(x)$ in Lemma 3.1, then, from Lemma 3.4, we get

$$
\mathcal{C}_{1}=\sup _{R>1} \int_{\mathbb{R}} \ell_{R}(x)(1+\varphi(x)) d x<\infty .
$$

Let $\Pi_{p} A_{t}(R)$ denote the projection of $A_{t}(R)$ on the $p$ th Wiener chaos, where $A_{t}(R)$ is defined in (1.3), that is,

$$
\Pi_{p} A_{t}(R)=I_{p}^{W}\left(\int_{-R}^{R} f_{t, x, p} d x\right) .
$$

In view of Theorem 1.4, we need first to establish the asymptotic covariance of each chaos of $\frac{1}{\sqrt{R}} A_{t}(R)$ and we know that

$$
\frac{1}{\sqrt{R}} A_{t}(R)=\sum_{p=1}^{\infty} \frac{1}{\sqrt{R}} \Pi_{p} A_{t}(R)=\sum_{p=1}^{\infty} I_{p}^{W}\left(\frac{1}{\sqrt{R}} \int_{-R}^{R} f_{t, x, p} d x\right) .
$$

Now we are ready to present the proof of Proposition 1.2.

Proof of Proposition 1.2 : In what follows, we only present the proof for the particular case where $t_{1}=t_{2}$, and the general case follows from the same arguments with solely notational modifications. Let us fix $t>0$ and break the proof into three steps.

Step 1: Proof of (1.6). Noting that $\Pi_{1} A_{t}(R)=I_{1}^{W}\left(\int_{-R}^{R} f_{t, x, 1} d x\right)$ and recalling Nualart and Zheng (2020a, page 32), we have

$$
\begin{aligned}
\operatorname{Var}\left[\Pi_{1}\left(A_{t}(R)\right)\right] & =\int_{-R}^{R} \int_{-R}^{R} d x d y\left\langle f_{s, x, 1}, f_{t, y, 1}\right\rangle_{\mathfrak{H}} \\
& =\int_{[0, t]^{2}} d r d v \gamma_{0}(r-v) \int_{\mathbb{R}} d \xi \int_{-R}^{R} \int_{-R}^{R} d x d y e^{-\mathbf{i}(x-y) \xi} \varphi(\xi) e^{-\frac{1}{2}(r+v) \xi^{2}} .
\end{aligned}
$$

We deduce from (3.15) that

$$
\frac{1}{2 R} \operatorname{Var}\left[\Pi_{1}\left(A_{t}(R)\right)\right]=2 \pi \int_{[0, t]^{2}} d r d v \gamma_{0}(r-v) \int_{\mathbb{R}} d \xi \ell_{R}(\xi) \varphi(\xi) e^{-\frac{1}{2}(r+v) \xi^{2}} .
$$

From Lemma 3.16 and using that $\gamma_{0}$ is locally integrable, we get for any $\varepsilon>0$,

$$
\frac{1}{2 R} \operatorname{Var}\left[\Pi_{1}\left(A_{t}(R)\right)\right] \leq C\left(\varepsilon+\frac{C(\varepsilon)}{R}\right)
$$

which implies $\lim _{R \rightarrow \infty} R^{-1} \operatorname{Var}\left[\Pi_{1}\left(A_{t}(R)\right)\right]=0$ in both cases (H1) and (H2).

Step 2: Proof of (1.5) and (1.7). Recall from Nualart and Zheng (2020a, page 31) that, for any $p \geq 2$,

$$
\begin{aligned}
\frac{1}{2 R} \operatorname{Var}\left[\Pi_{p}\left(A_{t}(R)\right)\right] & =\frac{1}{2 R} \int_{[-R, R]^{2}} d x d y \mathbb{E}\left[I_{p}^{W}\left(f_{t, x, p}\right) I_{p}^{W}\left(f_{t, y, p}\right)\right] \\
& =p ! \int_{\mathbb{R}} d z\left\langle f_{t, z, p}, f_{t, 0, p}\right\rangle_{\mathfrak{H} \otimes p} \frac{|[-R, R] \cap[-z-R,-z+R]|}{2 R}
\end{aligned}
$$


and

$$
\begin{aligned}
&\left\langle f_{t, z, p}, f_{t, 0, p}\right\rangle_{\mathfrak{H}^{\otimes p}}= \frac{1}{(p !)^{2}} \int_{[0, t]^{p} \times[0, t]^{p}} d \boldsymbol{s}_{\boldsymbol{p}} d \boldsymbol{r}_{\boldsymbol{p}} \prod_{j=1} \gamma_{0}\left(s_{j}-r_{j}\right) \int_{\mathbb{R}^{p}} \mu\left(d \boldsymbol{\xi}_{\boldsymbol{p}}\right) e^{-\mathbf{i} z \tau\left(\boldsymbol{\xi}_{\boldsymbol{p}}\right)} \\
& \times \mathbb{E}\left[\prod_{j=1}^{p} \exp \left(-\mathbf{i} B_{s_{j}} \xi_{j}\right)\right] \mathbb{E}\left[\prod_{j=1}^{p} \exp \left(-\mathbf{i} B_{r_{j}} \xi_{j}\right)\right]
\end{aligned}
$$

It is not difficult to deduce from Proposition 1.3 and the expression in (3.18) that

$$
(p !)^{2}\left\langle f_{t, z, p}, f_{t, 0, p}\right\rangle_{\mathfrak{H} \otimes p}=\mathbb{E}\left[\left(\mathcal{I}_{t, t}^{1,2}(z)\right)^{p}\right] .
$$

Then

$$
\frac{1}{2 R} \operatorname{Var}\left[\Pi_{p} A_{t}(R)\right]=\frac{1}{p !} \int_{\mathbb{R}} d z \mathbb{E}\left[\left(\mathcal{I}_{t, t}^{1,2}(z)\right)^{p}\right] \frac{|[-R, R] \cap[-z-R,-z+R]|}{2 R} .
$$

Because $|[-R, R] \cap[-z-R,-z+R]| /(2 R)$ converges to 1 as $R$ tends to infinity, the convergences (1.5) and (1.7) will be a consequence of

$$
\sum_{p=2}^{\infty} \frac{1}{p !} \int_{\mathbb{R}} d z \mathbb{E}\left[\left|\mathcal{I}_{t, t}^{1,2}(z)\right|^{p}\right]<\infty .
$$

In view of Fatou's lemma and taking into account that for any $z \in \mathbb{R}, \mathcal{I}_{t, t}^{1,2}(z)$ is the $L^{k}(\Omega)$-limit of $\mathcal{I}_{t, t, \varepsilon}^{1,2}(z)$ (for any $k \geq 2$ ) as $\varepsilon$ tends to zero, to show (3.19), it suffices to prove that

$$
\sup _{\varepsilon>0} \sum_{p=2}^{\infty} \frac{1}{p !} \int_{\mathbb{R}} d z \mathbb{E}\left[\left|\mathcal{I}_{t, t, \varepsilon}^{1,2}(z)\right|^{p}\right]<\infty
$$

From

$$
\sum_{p=2}^{\infty} \frac{|x|^{p}}{p !}=e^{|x|}-|x|-1 \leq\left(e^{x}+e^{-x}\right)-2=2 \sum_{n=1}^{\infty} \frac{x^{2 n}}{(2 n) !},
$$

we deduce that (3.20) holds true provided

$$
\sup _{\varepsilon>0} \sum_{n=1}^{\infty} \frac{1}{(2 n) !} \int_{\mathbb{R}} d z \mathbb{E}\left[\left(\mathcal{I}_{t, t, \varepsilon}^{1,2}(z)\right)^{2 n}\right]<\infty
$$

Next, let us prove (3.21). First consider the case (H1). Fix an even integer $p=2 n \geq 2$. For any $\varepsilon, a>0$, by Fubini's theorem,

$$
\begin{aligned}
& T_{\varepsilon, a}(p):=\int_{\mathbb{R}} \mathbb{E}\left[\left(\mathcal{I}_{t, t, \varepsilon}^{1,2}(z)\right)^{p}\right] \exp \left(-\frac{a}{2} z^{2}\right) d z \\
&=2 \pi \int_{[0, t]^{2 p}} \int_{\mathbb{R}^{p}} d \boldsymbol{\xi}_{\boldsymbol{p}} d \boldsymbol{s}_{\boldsymbol{p}} d \boldsymbol{r}_{\boldsymbol{p}}\left(\prod_{j=1}^{p} \gamma_{0}\left(s_{j}-r_{j}\right)\right)\left(\prod_{j=1}^{p} \varphi\left(\xi_{j}\right) e^{-\varepsilon \xi_{j}^{2}}\right) \\
& \times G\left(a, \tau\left(\boldsymbol{\xi}_{\boldsymbol{p}}\right)\right) \mathbb{E}\left[\prod_{j=1}^{p} \exp \left(-\mathbf{i} B_{s_{j}} \xi_{j}\right)\right] \mathbb{E}\left[\prod_{j=1}^{p} \exp \left(-\mathbf{i} B_{r_{j}} \xi_{j}\right)\right] .
\end{aligned}
$$


Note that $T_{\varepsilon, a}(p) \geq 0$ since $p$ is even. Using

$$
\mathbb{E}\left[\prod_{j=1}^{p} \exp \left(-\mathbf{i} B_{r_{j}} \xi_{j}\right)\right] \in(0,1]
$$

we can bound $T_{\varepsilon, a}(p)$ as follows:

$$
T_{\varepsilon, a}(p) \leq 2 \pi \Gamma_{t}^{p} \int_{\mathbb{R}^{p}} d \boldsymbol{\xi}_{\boldsymbol{p}} \int_{[0, t]^{p}} d \boldsymbol{s}_{\boldsymbol{p}}\left(\prod_{j=1}^{p} \varphi\left(\xi_{j}\right)\right) G\left(a, \tau\left(\boldsymbol{\xi}_{\boldsymbol{p}}\right)\right) \mathbb{E}\left[\prod_{j=1}^{p} \exp \left(-\mathbf{i} B_{s_{j}} \xi_{j}\right)\right]
$$

where the constant $\Gamma_{t}:=\int_{-t}^{t} \gamma_{0}(r) d r$ is finite for each $t>0$, since $\gamma_{0}$ is locally integrable. By the inequality (3.5) in Lemma 3.1,

$$
\sup _{a \in(0,1)} T_{\varepsilon, a}(p) \leq \sup _{a \in(0,1)} 2 \pi \Gamma_{t}^{p} K_{1, p}(G(a, \bullet), t) \leq \mathcal{C}_{0} t \pi \Gamma_{t}^{p} p !\left(8 \kappa_{0} C_{N}\right)^{p-1} e^{\frac{t D_{N}}{2 C_{N}}},
$$

where $\mathcal{C}_{0}$ is the constant defined in (3.14). Then, recalling $p=2 n \geq 2$ and letting $a \downarrow 0$, we obtain

$$
\frac{1}{(2 n) !} \int_{\mathbb{R}} \mathbb{E}\left[\left(\mathcal{I}_{t, t, \varepsilon}^{1,2}(z)\right)^{2 n}\right] d z \leq \mathcal{C}_{0} t \pi \Gamma_{t}^{2 n}\left(8 \kappa_{0} \Gamma_{t} C_{N}\right)^{2 n-1} e^{\frac{t D_{N}}{2 C_{N}}}
$$

which implies (3.21), provided we choose $N$ is such a way that $8 \kappa_{0} \Gamma_{t} C_{N}<1$.

Now we consider the case (H2) where $\gamma_{0}(t)=|t|^{2 H_{0}-2}$ and $\varphi(z)=|z|^{1-2 H_{1}}$ with $H_{0}>1 / 2>H_{1}$ and $H_{0}+H_{1}>3 / 4$. We begin with (3.22) and apply the embedding result (2.4) and Cauchy-Schwarz inequality to write for any $\varepsilon, a>0$ and any even $p=2 n \geq 2$,

$$
\begin{aligned}
T_{\varepsilon, a}(p) & \leq 2 \pi C_{H_{0}}^{p}\left[\int_{[0, t]^{p}} d \boldsymbol{s}_{\boldsymbol{p}}\left(\int_{\mathbb{R}^{p}} \mu\left(d \boldsymbol{\xi}_{\boldsymbol{p}}\right) G\left(a, \tau\left(\boldsymbol{\xi}_{\boldsymbol{p}}\right)\right) e^{-\operatorname{Var} \sum_{j=1}^{p} B_{s_{j}} \xi_{j}}\right)^{\frac{1}{2 H_{0}}}\right]^{2 H_{0}} \\
& =2 \pi C_{H_{0}}^{p}\left[K_{2, p}(G(a, \bullet), t)\right]^{2 H_{0}}
\end{aligned}
$$

where $K_{2, p}(G(a, \bullet), t)$ has been defined in (3.2). Then, applying inequality (3.8) of Lemma 3.1 leads to

$$
T_{\varepsilon, a}(p) \leq 2 \pi C_{H_{0}}^{p}\left(p ! \mathcal{C}_{0}\right)^{2 H_{0}} \frac{C_{1}^{2 H_{0} p}\left(t^{2 H_{0} p \mathfrak{h}+1-H_{1}} \vee t^{2 H_{0} p \mathfrak{h}+\frac{1}{2}}\right)}{\Gamma\left(p \mathfrak{h}+\frac{1-H_{1}}{2 H_{0}}\right)^{2 H_{0}} \wedge \Gamma\left(p \mathfrak{h}+\frac{1}{4 H_{0}}\right)^{2 H_{0}}},
$$

for $\varepsilon>0, a \in(0,1)$ and any even integer $p=2 n \geq 2$, where $\mathfrak{h}=\frac{2 H_{0}+H_{1}-1}{2 H_{0}}>\frac{1}{4}$ and with $\mathcal{C}_{0}$ being the constant defined in (3.14). Thus, letting $a \downarrow 0$, we have for $n \geq 1$

$$
\frac{1}{(2 n) !} \int_{\mathbb{R}} \mathbb{E}\left[\left(\mathcal{I}_{t, t, \varepsilon}^{1,2}(z)\right)^{2 n}\right] d z \leq \frac{C_{2}^{2 n}((2 n) !)^{2 H_{0}-1}\left(t^{4 H_{0} n \mathfrak{h}+1-H_{1}} \vee t^{4 H_{0} n \mathfrak{h}+\frac{1}{2}}\right)}{\Gamma\left(2 n \mathfrak{h}+\frac{1-H_{1}}{2 H_{0}}\right)^{2 H_{0}} \wedge \Gamma\left(2 n \mathfrak{h}+\frac{1}{4 H_{0}}\right)^{2 H_{0}}}
$$

for some constant $C_{2}$, which depends only on $H_{0}$ and $H_{1}$. Notice that the denominator in the right-hand side of (3.24) behaves as $[(2 n) !]^{2 H_{0} \mathfrak{h}}$, as $n \rightarrow \infty$, and $2 H_{0} \mathfrak{h}=2 H_{0}+H_{1}-1$. Thus, the estimate (3.24) implies (3.21) in the case (H2). Hence the proof of Proposition 1.2 is completed now. 
Remark 3.6. We observe that $\frac{1}{2 R} \operatorname{Var}\left(A_{t}(R)\right)$ is equal to

$$
\frac{1}{2 R} \operatorname{Var}\left[\int_{-R}^{R}(u(t, x)-1) d x\right]=\frac{1}{2 R} \int_{[-R, R]^{2}}(\mathbb{E}[u(t, x) u(t, y)]-1) d x d y,
$$

which in view of Proposition 1.3, is equal to

$$
\frac{1}{2 R} \int_{[-R, R]^{2}} \mathbb{E}\left[\left(e^{\mathcal{I}_{t, t}^{1,2}(x-y)}-1\right)\right] d x d y=\int_{\mathbb{R}} \frac{|[z-R, z+R] \cap[-R, R]|}{2 R} \mathbb{E}\left[e^{\mathcal{I}_{t, t}^{1,2}(z)}-1\right] d z .
$$

As $R \rightarrow \infty$, this converges to

$$
\int_{\mathbb{R}} \mathbb{E}\left[e^{\mathcal{I}_{t, t}^{1,2}(z)}-1\right] d z=\int_{\mathbb{R}} \sum_{p=2}^{\infty} \frac{1}{p !} \mathbb{E}\left[\left(\mathcal{I}_{t, t}^{1,2}(z)\right)^{p}\right] d z,
$$

since $\mathbb{E}\left[\mathcal{I}_{t, t}^{1,2}(z)\right]=\lim _{\varepsilon \downarrow 0} \mathbb{E}\left[\mathcal{I}_{t, t, \varepsilon}^{1,2}(z)\right]=0$, based on Step 1 in the proof of Proposition 1.2. The above limit is exactly the one in (1.5).

\section{Convergence of finite-dimensional distributions}

To apply the multivariate chaotic CLT (Theorem 1.4), we need to show the convergence of finite-dimensional distributions. There are four conditions in this theorem that we need to check. In fact, we only need to verify the condition (c), as the other conditions are satisfied as a consequence of our estimates in the previous section. We refer readers to Nualart and Zheng (2020a) for similar arguments.

We can write

$$
\frac{1}{\sqrt{R}} A_{t}(R)=\sum_{p=1}^{\infty} I_{p}^{W}\left(g_{p, R}(t)\right)
$$

where

$$
g_{p, R}(t):=\frac{1}{\sqrt{R}} \int_{-R}^{R} f_{t, x, p} d x .
$$

Proposition 4.1. For each integer $p \geq 2$ and each integer $1 \leq r \leq p-1$, we have

$$
\left\|g_{p, R}(t) \otimes_{r} g_{p, R}(t)\right\|_{\mathfrak{H}^{\otimes 2 p-2 r}} \stackrel{R \rightarrow \infty}{\longrightarrow} 0 .
$$

Proof: Along the proof $C$ will be a generic constant that may very form line to line. We put

$$
\mathfrak{f}\left(\boldsymbol{s}_{\boldsymbol{p}}, \boldsymbol{y}_{\boldsymbol{p}}\right)=f_{t, 0, p}\left(\boldsymbol{s}_{\boldsymbol{p}}, \boldsymbol{y}_{\boldsymbol{p}}\right)
$$

and recall that, with $B$ a real-valued standard Brownian motion on $\mathbb{R}$,

$$
(\mathcal{F} \mathfrak{f})\left(\boldsymbol{s}_{\boldsymbol{p}}, \boldsymbol{\xi}_{\boldsymbol{p}}\right)=\left(\mathcal{F} f_{t, 0, p}\right)\left(\boldsymbol{s}_{\boldsymbol{p}}, \boldsymbol{\xi}_{\boldsymbol{p}}\right)=\frac{1}{p !} \mathbb{E}\left[\exp \left(-\mathbf{i} \sum_{j=1}^{p} B_{s_{j}} \xi_{j}\right)\right],
$$

where $\mathcal{F} \mathfrak{f}$ stands for the Fourier transform with respect to the spatial variables. As a consequence, $(\mathcal{F} \mathfrak{f})\left(\boldsymbol{s}_{\boldsymbol{p}}, \boldsymbol{\xi}_{\boldsymbol{p}}\right)$ is a positive, bounded and uniformly continuous function in $\xi_{p}$. 
Now we write, with the notation $d \ell^{4 p}=d \boldsymbol{s}_{\boldsymbol{r}} d \widetilde{\boldsymbol{s}}_{\boldsymbol{r}} d \boldsymbol{v}_{\boldsymbol{r}} d \widetilde{\boldsymbol{v}}_{\boldsymbol{r}} d \boldsymbol{t}_{\boldsymbol{p}-\boldsymbol{r}} d \widetilde{\boldsymbol{t}}_{\boldsymbol{p}-\boldsymbol{r}} d \boldsymbol{w}_{\boldsymbol{p}-\boldsymbol{r}} d \widetilde{\boldsymbol{w}}_{\boldsymbol{p}-\boldsymbol{r}}$ for the Lebesgue measure on $[0, t]^{4 p}$,

$$
\begin{gathered}
\left\|g_{p, R}(t) \otimes_{r} g_{p, R}(t)\right\|_{\mathfrak{H}^{\otimes(2 p-2 r)}}^{2}=(2 \pi)^{2} \int_{[0, t]^{4 p}} d \ell^{4 p}\left(\prod_{i=1}^{r} \gamma_{0}\left(s_{i}-\widetilde{s}_{i}\right) \gamma_{0}\left(v_{i}-\widetilde{v}_{i}\right)\right) \\
\times\left(\prod_{j=1}^{p-r} \gamma_{0}\left(t_{j}-\widetilde{t}_{j}\right) \gamma_{0}\left(w_{j}-\widetilde{w}_{j}\right)\right) \mathcal{J}_{R},
\end{gathered}
$$

with $\mathcal{J}_{R}=\mathcal{J}_{R}\left(\boldsymbol{s}_{\boldsymbol{r}}, \tilde{\boldsymbol{s}}_{\boldsymbol{r}}, \boldsymbol{v}_{\boldsymbol{r}}, \widetilde{\boldsymbol{v}}_{\boldsymbol{r}}, \boldsymbol{t}_{\boldsymbol{p}-\boldsymbol{r}}, \widetilde{\boldsymbol{t}}_{\boldsymbol{p}-\boldsymbol{r}}, \boldsymbol{w}_{\boldsymbol{p}-\boldsymbol{r}}, \widetilde{\boldsymbol{w}}_{\boldsymbol{p}-\boldsymbol{r}}\right)$ given by

$$
\begin{aligned}
\mathcal{J}_{R}= & \int_{\mathbb{R}^{2 p}} \mu\left(d \boldsymbol{\xi}_{\boldsymbol{r}}\right) \mu\left(d \widetilde{\boldsymbol{\xi}}_{\boldsymbol{r}}\right) \mu\left(d \boldsymbol{\eta}_{\boldsymbol{p}-\boldsymbol{r}}\right) \mu\left(d \widetilde{\boldsymbol{\eta}}_{\boldsymbol{p}-\boldsymbol{r}}\right) \\
& \times(\mathcal{F} \mathfrak{f})\left(\boldsymbol{s}_{\boldsymbol{r}}, \boldsymbol{t}_{\boldsymbol{p}-\boldsymbol{r}}, \boldsymbol{\eta}_{\boldsymbol{p}-\boldsymbol{r}}, \boldsymbol{\xi}_{\boldsymbol{r}}\right)(\mathcal{F} \mathfrak{f})\left(\widetilde{\boldsymbol{s}}_{\boldsymbol{r}}, \boldsymbol{w}_{\boldsymbol{p}-\boldsymbol{r}}, \widetilde{\boldsymbol{\eta}}_{\boldsymbol{p}-\boldsymbol{r}},-\boldsymbol{\xi}_{\boldsymbol{r}}\right)|a+b|^{-1 / 2}|a-\widetilde{b}|^{-1 / 2} \\
& \times(\mathcal{F} \mathfrak{f})\left(\boldsymbol{v}_{\boldsymbol{r}}, \widetilde{\boldsymbol{t}}_{\boldsymbol{p}-\boldsymbol{r}},-\boldsymbol{\eta}_{\boldsymbol{p}-\boldsymbol{r}}, \widetilde{\boldsymbol{\xi}}_{\boldsymbol{r}}\right)(\mathcal{F} \mathfrak{f})\left(\widetilde{\boldsymbol{v}}_{\boldsymbol{r}}, \widetilde{\boldsymbol{w}}_{\boldsymbol{p}-\boldsymbol{r}},-\widetilde{\boldsymbol{\eta}}_{\boldsymbol{p}-\boldsymbol{r}},-\widetilde{\boldsymbol{\xi}}_{\boldsymbol{r}}\right)|\widetilde{a}-b|^{-1 / 2}|\widetilde{a}+\widetilde{b}|^{-1 / 2} \\
& \times J_{1 / 2}(R|a+b|) J_{1 / 2}(R|a-\widetilde{b}|) J_{1 / 2}(R|\widetilde{a}-b|) J_{1 / 2}(R|\widetilde{a}+\widetilde{b}|),
\end{aligned}
$$

where we use the following short-hand notation $a=\tau\left(\boldsymbol{\xi}_{\boldsymbol{r}}\right), b=\tau\left(\boldsymbol{\eta}_{\boldsymbol{p}-\boldsymbol{r}}\right), \widetilde{a}=\tau\left(\widetilde{\boldsymbol{\xi}}_{\boldsymbol{r}}\right)$, $\widetilde{b}=\tau\left(\widetilde{\boldsymbol{\eta}}_{\boldsymbol{p}-\boldsymbol{r}}\right)$ and $J_{1 / 2}(x)=\sqrt{\frac{2}{\pi}} \frac{\sin (x)}{\sqrt{x}}, x \in \mathbb{R}_{+}$is the Bessel function of first kind with order $1 / 2$. This is obtained in the same way as in Nualart and Zheng (2020a) and we refer readers to this reference for more details.

Now we decompose the integral in the spatial variable into two parts, and we write for any given $\delta>0, \mathcal{J}_{R}=\mathcal{J}_{1, R, \delta}+\mathcal{J}_{2, R, \delta}:=\int_{\mathbb{R}^{2 p}} \mathbf{1}_{\{|a+b| \geq \delta\}}+\int_{\mathbb{R}^{2 p}} \mathbf{1}_{\{|a+b|<\delta\}}$. This leads to the decomposition

$$
\left\|g_{p, R}(t) \otimes_{r} g_{p, R}(t)\right\|_{\mathfrak{H}^{\otimes(2 p-2 r)}}^{2}=(2 \pi)^{2}\left(\mathcal{Y}_{1, R, \delta}+\mathcal{Y}_{2, R, \delta}\right),
$$

where, for $k=1,2$,

$\mathcal{Y}_{k, R, \delta}$

$$
:=\int_{[0, t]^{4 p}} d \ell^{4 p}\left(\prod_{i=1}^{r} \gamma_{0}\left(s_{i}-\widetilde{s}_{i}\right) \gamma_{0}\left(v_{i}-\widetilde{v}_{i}\right)\right) \times\left(\prod_{j=1}^{p-r} \gamma_{0}\left(t_{j}-\widetilde{t}_{j}\right) \gamma_{0}\left(w_{j}-\widetilde{w}_{j}\right)\right) \mathcal{J}_{k, R, \delta} .
$$

The proof will be done in two steps.

Step 1: We will show that for any fixed $\delta>0, \mathcal{Y}_{1, R, \delta}$ tends to zero as $R \rightarrow \infty$. First we apply Cauchy-Schwarz inequality several times to get

$$
\begin{aligned}
& \mathcal{J}_{1, R, \delta} \leq 4\left(\int_{\left\{\left|\tau\left(\boldsymbol{\xi}_{\boldsymbol{p}}\right)\right| \geq \delta\right\}} \ell_{R}\left(\tau\left(\boldsymbol{\xi}_{\boldsymbol{p}}\right)\right)|\mathcal{F} \mathfrak{f}|^{2}\left(\boldsymbol{s}_{\boldsymbol{r}}, \boldsymbol{t}_{\boldsymbol{p}-\boldsymbol{r}}, \boldsymbol{\xi}_{\boldsymbol{p}}\right) \mu\left(d \boldsymbol{\xi}_{\boldsymbol{p}}\right)\right)^{1 / 2} \\
& \times\left(\int_{\mathbb{R}^{p}} \ell_{R}\left(\tau\left(\boldsymbol{\xi}_{\boldsymbol{p}}\right)\right)|\mathcal{F} \mathfrak{f}|^{2}\left(\widetilde{\boldsymbol{v}}_{\boldsymbol{r}}, \widetilde{\boldsymbol{w}}_{\boldsymbol{p}-\boldsymbol{r}}, \boldsymbol{\xi}_{\boldsymbol{p}}\right) \mu\left(d \boldsymbol{\xi}_{\boldsymbol{p}}\right)\right)^{1 / 2}\left(\int_{\mathbb{R}^{p}} \mu\left(d \boldsymbol{\xi}_{\boldsymbol{p}}\right) \ell_{R}\left(\tau\left(\boldsymbol{\xi}_{\boldsymbol{p}}\right)\right)\right. \\
&\left.\quad \times|\mathcal{F} \mathfrak{f}|^{2}\left(\widetilde{\boldsymbol{s}}_{\boldsymbol{r}}, \boldsymbol{w}_{\boldsymbol{p}-\boldsymbol{r}}, \boldsymbol{\xi}_{\boldsymbol{p}}\right)\right)^{1 / 2}\left(\int_{\mathbb{R}^{p}} \ell_{R}\left(\tau\left(\boldsymbol{\xi}_{\boldsymbol{p}}\right)\right)|\mathcal{F} \mathfrak{f}|^{2}\left(\boldsymbol{v}_{\boldsymbol{r}}, \widetilde{\boldsymbol{t}}_{\boldsymbol{p}-\boldsymbol{r}}, \boldsymbol{\xi}_{\boldsymbol{p}}\right) \mu\left(d \boldsymbol{\xi}_{\boldsymbol{p}}\right)\right)^{1 / 2},
\end{aligned}
$$

where $\ell_{R}(x)=\frac{1}{2}|x|^{-1} J_{1 / 2}^{2}(R|x|)$ is introduced in (3.15). We will prove separately for cases (H1) and (H2) that $\mathcal{Y}_{1, R, \delta} \rightarrow 0$ as $R \rightarrow \infty$. 
Proof of $\mathcal{Y}_{1, R, \delta} \stackrel{R \rightarrow \infty}{\longrightarrow} 0$ under (H1): By Cauchy-Schwarz inequality again applied to the integration in time, we get

$$
\begin{aligned}
\mathcal{Y}_{1, R, \delta} \leq 4\left\{\int_{[0, t]^{4 p}} d \ell^{4 p}\left(\prod_{i=1}^{r} \gamma_{0}\left(s_{i}-\widetilde{s}_{i}\right) \gamma_{0}\left(v_{i}-\widetilde{v}_{i}\right)\right)\left(\prod_{j=1}^{p-r} \gamma_{0}\left(t_{j}-\widetilde{t}_{j}\right) \gamma_{0}\left(w_{j}-\widetilde{w}_{j}\right)\right)\right. \\
\times\left(\int_{\mathbb{R}^{p}} \ell_{R}\left(\tau\left(\boldsymbol{\xi}_{\boldsymbol{p}}\right)\right)|\mathcal{F} \mathfrak{f}|^{2}\left(\widetilde{\boldsymbol{v}}_{\boldsymbol{r}}, \widetilde{\boldsymbol{w}}_{\boldsymbol{p}-\boldsymbol{r}}, \boldsymbol{\xi}_{\boldsymbol{p}}\right) \mu\left(d \boldsymbol{\xi}_{\boldsymbol{p}}\right)\right) \\
\left.\times \int_{\left\{\left|\tau\left(\boldsymbol{\xi}_{\boldsymbol{p}}\right)\right| \geq \delta\right\}} \ell_{R}\left(\tau\left(\boldsymbol{\xi}_{\boldsymbol{p}}\right)\right)|\mathcal{F} \mathfrak{f}|^{2}\left(\boldsymbol{s}_{\boldsymbol{r}}, \boldsymbol{t}_{\boldsymbol{p}-\boldsymbol{r}}, \boldsymbol{\xi}_{\boldsymbol{p}}\right) \mu\left(d \boldsymbol{\xi}_{\boldsymbol{p}}\right)\right\}^{1 / 2} \\
\times\left\{\int_{[0, t]^{4 p}} d \ell^{4 p}\left(\prod_{i=1}^{r} \gamma_{0}\left(s_{i}-\widetilde{s}_{i}\right) \gamma_{0}\left(v_{i}-\widetilde{v}_{i}\right)\right)\left(\prod_{j=1}^{p-r} \gamma_{0}\left(t_{j}-\widetilde{t}_{j}\right) \gamma_{0}\left(w_{j}-\widetilde{w}_{j}\right)\right)\right. \\
\times\left(\int_{\mathbb{R}^{p}} \ell_{R}\left(\tau\left(\boldsymbol{\xi}_{\boldsymbol{p}}\right)\right)|\mathcal{F} \mathfrak{f}|^{2}\left(\widetilde{\boldsymbol{s}}_{\boldsymbol{r}}, \boldsymbol{w}_{\boldsymbol{p}-\boldsymbol{r}}, \boldsymbol{\xi}_{\boldsymbol{p}}\right) \mu\left(d \boldsymbol{\xi}_{\boldsymbol{p}}\right)\right) \\
\left.\times \int_{\mathbb{R}^{p}} \ell_{R}\left(\tau\left(\boldsymbol{\xi}_{\boldsymbol{p}}\right)\right)|\mathcal{F} \mathfrak{f}|^{2}\left(\boldsymbol{v}_{\boldsymbol{r}}, \widetilde{\boldsymbol{t}}_{\boldsymbol{p}-\boldsymbol{r}}, \boldsymbol{\xi}_{\boldsymbol{p}}\right) \mu\left(d \boldsymbol{\xi}_{\boldsymbol{p}}\right)\right\}^{1 / 2}=: 4 V_{1, R, \delta}^{1 / 2} V_{2, R}^{1 / 2} .
\end{aligned}
$$

For the term $V_{1, R, \delta}$, we have the estimate

$$
\begin{aligned}
V_{1, R, \delta} & \leq \Gamma_{t}^{2 p}\left[\int_{[0, t]^{p}} d \boldsymbol{s}_{\boldsymbol{p}} \int_{\left\{\left|\tau\left(\boldsymbol{\xi}_{\boldsymbol{p}}\right)\right| \geq \delta\right\}} \ell_{R}\left(\tau\left(\boldsymbol{\xi}_{\boldsymbol{p}}\right)\right)|\mathcal{F} \mathfrak{f}|^{2}\left(\boldsymbol{s}_{\boldsymbol{p}}, \boldsymbol{\xi}_{\boldsymbol{p}}\right) \mu\left(d \boldsymbol{\xi}_{\boldsymbol{p}}\right)\right] \\
& \times\left(\int_{[0, t]^{p}} d \boldsymbol{t}_{\boldsymbol{p}} \int_{\mathbb{R}^{p}} \ell_{R}\left(\tau\left(\boldsymbol{\xi}_{\boldsymbol{p}}\right)\right)|\mathcal{F} \mathfrak{f}|^{2}\left(\boldsymbol{t}_{\boldsymbol{p}}, \boldsymbol{\xi}_{\boldsymbol{p}}\right) \mu\left(d \boldsymbol{\xi}_{\boldsymbol{p}}\right)\right)=: \Gamma_{t}^{2 p} V_{11, R, \delta} V_{12, R},
\end{aligned}
$$

where we recall that $\Gamma_{t}=\int_{-t}^{t} \gamma_{0}(s) d s$. We will prove that $V_{12, R}$ is uniformly bounded and $V_{11, R, \delta}$ vanishes asymptotically as $R \rightarrow \infty$. In view of (4.1), making the change of variables $t_{j}=t-s_{j}$ and $\eta_{j}=\xi_{1}+\cdots+\xi_{j}$ for each $j=1, \ldots, p$, with $\eta_{0}=0$, we obtain, in view of (3.3),

$$
\begin{aligned}
V_{12, R} & =\frac{1}{p !} \int_{\Delta_{p}(t)} d \boldsymbol{s}_{\boldsymbol{p}} \int_{\mathbb{R}^{p}} \mu\left(d \boldsymbol{\xi}_{\boldsymbol{p}}\right) \ell_{R}\left(\tau\left(\boldsymbol{\xi}_{\boldsymbol{p}}\right)\right) \exp \left(-\sum_{j=1}^{p}\left(s_{j}-s_{j+1}\right)\left(\xi_{1}+\cdots+\xi_{j}\right)^{2}\right) \\
& =\frac{1}{p !} \int_{\mathbb{R}} d \eta_{p} \ell_{R}\left(\eta_{p}\right) \int_{\mathbb{R}^{p-1}} d \boldsymbol{\eta}_{\boldsymbol{p}-\mathbf{1}} \int_{\operatorname{SIM}_{p}(t)} d \boldsymbol{w}_{\boldsymbol{p}} \prod_{j=1}^{p} e^{-w_{j} \eta_{j}^{2}} \varphi\left(\eta_{j}-\eta_{j-1}\right) \\
& =K_{1, p}\left(\ell_{R}, t\right) .
\end{aligned}
$$

By inequality (3.5) in Lemma 3.1 and (3.17), this implies

$$
\sup _{R>1} V_{12, R}<\infty \text {. }
$$

In the same way, we have, for any $\varepsilon>0$, using (3.5) and (3.16),

$$
\begin{aligned}
V_{11, R, \delta} & \leq K_{1, p}\left(\ell_{R} \mathbf{1}_{(-\delta, \delta)^{c}}, t\right) \leq C \int_{\{|\eta| \geq \delta\}} d \eta \ell_{R}(\eta)(1+\varphi(\eta)) \\
& \leq C \int_{\{|\eta| \geq R \delta\}} d \eta \frac{\sin ^{2}(\eta)}{\pi \eta^{2}}+C\left(\varepsilon+\frac{C(\varepsilon)}{R}\right)
\end{aligned}
$$


for some constant $C>0$. So, $\lim _{R \rightarrow \infty} V_{11, R, \delta}=0$ and, therefore, from (4.4), (4.2) and (4.3) we have proved that

$$
V_{1, R, \delta} \stackrel{R \rightarrow \infty}{\longrightarrow} 0 .
$$

For the term $V_{2, R}$, note that $V_{2, R} \leq \Gamma_{t}^{2 p} V_{12, R}^{2}$, so we have the uniform boundedness of $V_{2, R}$ over $R>1$. Thus, we completed the proof of $\mathcal{Y}_{1, R, \delta} \stackrel{R \rightarrow \infty}{\longrightarrow} 0$ under (H1).

Proof of $\mathcal{Y}_{1, R, \delta} \stackrel{R \rightarrow \infty}{\longrightarrow} 0$ under (H2): Using the embedding result (2.4) and Cauchy-Schwarz inequality, we can write

$$
\begin{aligned}
& \mathcal{Y}_{1, R, \delta} \leq C_{H_{0}}^{p}\left[\int_{[0, t]^{2 p}} d \boldsymbol{s}_{\boldsymbol{p}} d \boldsymbol{t}_{\boldsymbol{p}}\left(\int_{\left\{\left|\tau\left(\boldsymbol{\xi}_{\boldsymbol{p}}\right)\right| \geq \delta\right\}} \ell_{R}\left(\tau\left(\boldsymbol{\xi}_{\boldsymbol{p}}\right)\right)|\mathcal{F} \mathfrak{f}|^{2}\left(\boldsymbol{t}_{\boldsymbol{p}}, \boldsymbol{\xi}_{\boldsymbol{p}}\right) \mu\left(d \boldsymbol{\xi}_{\boldsymbol{p}}\right)\right)^{\frac{1}{2 H_{0}}}\right. \\
&\left.\times\left(\int_{\mathbb{R}^{p}} \ell_{R}\left(\tau\left(\boldsymbol{\xi}_{\boldsymbol{p}}\right)\right)|\mathcal{F} \mathfrak{f}|^{2}\left(\boldsymbol{s}_{\boldsymbol{p}}, \boldsymbol{\xi}_{\boldsymbol{p}}\right) \mu\left(d \boldsymbol{\xi}_{\boldsymbol{p}}\right)\right)^{\frac{1}{2 H_{0}}}\right]^{H_{0}} \\
& \times\left[\int_{[0, t]^{2 p}} d \boldsymbol{s}_{\boldsymbol{p}} d \boldsymbol{t}_{\boldsymbol{p}}\left(\int_{\mathbb{R}^{p}} \ell_{R}\left(\tau\left(\boldsymbol{\xi}_{\boldsymbol{p}}\right)\right)|\mathcal{F} \mathfrak{f}|^{2}\left(\boldsymbol{t}_{\boldsymbol{p}}, \boldsymbol{\xi}_{\boldsymbol{p}}\right) \mu\left(d \boldsymbol{\xi}_{\boldsymbol{p}}\right)\right)^{\frac{1}{2 H_{0}}}\right. \\
&\left.\times\left(\int_{\mathbb{R}^{p}} \ell_{R}\left(\tau\left(\boldsymbol{\xi}_{\boldsymbol{p}}\right)\right)|\mathcal{F} \mathfrak{f}|^{2}\left(\boldsymbol{s}_{\boldsymbol{p}}, \boldsymbol{\xi}_{\boldsymbol{p}}\right) \mu\left(d \boldsymbol{\xi}_{\boldsymbol{p}}\right)\right)^{\frac{1}{2 H_{0}}}\right]^{H_{0}}=C_{H_{0}}^{p} T_{1, R, \delta}^{H_{0}} T_{2, R}^{3 H_{0}},
\end{aligned}
$$

where

$$
\begin{aligned}
T_{1, R, \delta} & :=\int_{[0, t]^{p}} d \boldsymbol{t}_{\boldsymbol{p}}\left(\int_{\left\{\left|\tau\left(\boldsymbol{\xi}_{\boldsymbol{p}}\right)\right| \geq \delta\right\}} \ell_{R}\left(\tau\left(\boldsymbol{\xi}_{\boldsymbol{p}}\right)\right)|\mathcal{F} \mathfrak{f}|^{2}\left(\boldsymbol{t}_{\boldsymbol{p}}, \boldsymbol{\xi}_{\boldsymbol{p}}\right), \mu\left(d \boldsymbol{\xi}_{\boldsymbol{p}}\right)\right)^{\frac{1}{2 H_{0}}} \text { and } \\
T_{2, R} & :=\int_{[0, t]^{p}} d \boldsymbol{t}_{\boldsymbol{p}}\left(\int_{\mathbb{R}^{p}} \ell_{R}\left(\tau\left(\boldsymbol{\xi}_{\boldsymbol{p}}\right)\right)|\mathcal{F} \mathfrak{f}|^{2}\left(\boldsymbol{t}_{\boldsymbol{p}}, \boldsymbol{\xi}_{\boldsymbol{p}}\right) \mu\left(d \boldsymbol{\xi}_{\boldsymbol{p}}\right)\right)^{\frac{1}{2 H_{0}}} .
\end{aligned}
$$

From (4.1), a change of variables and inequalities (3.8) and (3.17), we can easily get

$$
\begin{aligned}
T_{2, R}= & (p !)^{-\frac{1}{H_{0}}} \int_{[0, t]^{p}} d \boldsymbol{s}_{\boldsymbol{p}}\left(\int_{\mathbb{R}^{p}} \mu\left(d \boldsymbol{\xi}_{\boldsymbol{p}}\right) \ell_{R}\left(\tau\left(\boldsymbol{\xi}_{\boldsymbol{p}}\right)\right) \exp \left[-\operatorname{Var} \sum_{j=1}^{p} B_{s_{j}} \xi_{j}\right]\right)^{\frac{1}{2 H_{0}}} \\
& =(p !)^{-\frac{1}{H_{0}}} K_{2, p}\left(\ell_{R}, t\right) \leq C
\end{aligned}
$$

for all $R>1$. The term $T_{1, R, \delta}$ can be estimated as follows

$$
\begin{aligned}
T_{1, R, \delta} & =(p !)^{-\frac{1}{H_{0}}} \int_{[0, t]^{p}} d \boldsymbol{s}_{\boldsymbol{p}}\left(\int_{\left\{\left|\eta_{p}\right| \geq \delta\right\}} \mu\left(d \boldsymbol{\xi}_{\boldsymbol{p}}\right) \ell_{R}\left(\tau\left(\boldsymbol{\xi}_{\boldsymbol{p}}\right)\right) \exp \left[-\operatorname{Var} \sum_{j=1}^{p} B_{s_{j}} \xi_{j}\right]\right)^{\frac{1}{2 H_{0}}} \\
& =(p !)^{-\frac{1}{H_{0}}} K_{2, p}\left(\ell_{R} \mathbf{1}_{(-\delta, \delta)^{c}}, t\right) \leq C \int_{\{|\eta| \geq \delta\}} d \eta \ell_{R}(\eta)\left(1+|\eta|^{1-2 H_{1}}\right),
\end{aligned}
$$

which converges to zero as $R \rightarrow \infty$ as we have already noted. Therefore, combining the calculations on $T_{1, R, \delta}$ and $T_{2, R}$, we show that $\mathcal{Y}_{1, R, \delta} \stackrel{R \rightarrow \infty}{\longrightarrow} 0$ under (H2).

Step 2: We will show that

$$
\lim _{\delta \rightarrow 0} \limsup _{R \rightarrow \infty} \mathcal{Y}_{2, R, \delta}=0 .
$$


Using Cauchy-Schwarz multiple times and changing of variables, we obtain

$$
\begin{aligned}
& \mathcal{J}_{2, R, \delta} \leq 4 \int_{\{|a+b|<\delta\}} \mu\left(d \boldsymbol{\xi}_{\boldsymbol{r}}\right) \mu\left(d \boldsymbol{\eta}_{\boldsymbol{p}-\boldsymbol{r}}\right) \sqrt{\ell_{R}(a+b)} \mathcal{F} \mathfrak{f}\left(\boldsymbol{s}_{\boldsymbol{r}}, \boldsymbol{t}_{\boldsymbol{p}-\boldsymbol{r}}, \boldsymbol{\eta}_{\boldsymbol{p}-\boldsymbol{r}}, \boldsymbol{\xi}_{\boldsymbol{r}}\right) \\
& \times\left(\int_{\mathbb{R}^{p}} \mu\left(d \widetilde{\boldsymbol{\xi}}_{\boldsymbol{r}}\right) \mu\left(d \widetilde{\boldsymbol{\eta}}_{\boldsymbol{p}-\boldsymbol{r}}\right) \ell_{R}(\widetilde{a}+\widetilde{b})|\mathcal{F} \mathfrak{f}|^{2}\left(\widetilde{\boldsymbol{v}}_{\boldsymbol{r}}, \widetilde{\boldsymbol{w}}_{\boldsymbol{p}-\boldsymbol{r}}, \widetilde{\boldsymbol{\eta}}_{\boldsymbol{p}-\boldsymbol{r}}, \widetilde{\boldsymbol{\xi}}_{\boldsymbol{r}}\right)\right)^{1 / 2}\left[\int_{\mathbb{R}^{p}} \mu\left(d \widetilde{\boldsymbol{\xi}}_{\boldsymbol{r}}\right)\right. \\
& \left.\times \mu\left(d \widetilde{\boldsymbol{\eta}}_{\boldsymbol{p}-\boldsymbol{r}}\right) \ell_{R}(\widetilde{a}+b) \ell_{R}(a+\widetilde{b})|\mathcal{F} \mathfrak{f}|^{2}\left(\boldsymbol{v}_{\boldsymbol{r}}, \widetilde{\boldsymbol{t}}_{\boldsymbol{p}-\boldsymbol{r}}, \boldsymbol{\eta}_{\boldsymbol{p}-\boldsymbol{r}}, \widetilde{\boldsymbol{\xi}}_{\boldsymbol{r}}\right)|\mathcal{F} \mathfrak{F}|^{2}\left(\widetilde{\boldsymbol{s}}_{\boldsymbol{r}}, \boldsymbol{w}_{\boldsymbol{p}-\boldsymbol{r}}, \widetilde{\boldsymbol{\eta}}_{\boldsymbol{p}-\boldsymbol{r}}, \boldsymbol{\xi}_{\boldsymbol{r}}\right)\right]^{1 / 2} \\
& \leq 4\left[\left(\int_{\mathbb{R}^{p}} \mu\left(d \widetilde{\boldsymbol{\xi}}_{\boldsymbol{p}}\right) \ell_{R}\left(\tau\left(\tilde{\boldsymbol{\xi}}_{\boldsymbol{p}}\right)\right)|\mathcal{F} \mathfrak{f}|^{2}\left(\widetilde{\boldsymbol{v}}_{\boldsymbol{r}}, \widetilde{\boldsymbol{w}}_{\boldsymbol{p}-\boldsymbol{r}}, \widetilde{\boldsymbol{\xi}}_{\boldsymbol{p}}\right)\right)\right. \\
& \left.\times\left(\int_{\left\{\left|\tau\left(\boldsymbol{\xi}_{\boldsymbol{p}}\right)\right|<\delta\right\}} \mu\left(d \boldsymbol{\xi}_{\boldsymbol{p}}\right) \ell_{R}\left(\tau\left(\boldsymbol{\xi}_{\boldsymbol{p}}\right)\right)|\mathcal{F} \mathfrak{f}|^{2}\left(\boldsymbol{s}_{\boldsymbol{r}}, \boldsymbol{t}_{\boldsymbol{p}-\boldsymbol{r}}, \boldsymbol{\xi}_{\boldsymbol{p}}\right)\right)\right]^{1 / 2} \\
& \times\left[\int_{\{|a+b|<\delta\} \times \mathbb{R}^{p}} \mu\left(d \boldsymbol{\xi}_{\boldsymbol{r}}\right) \mu\left(d \boldsymbol{\eta}_{\boldsymbol{p}-\boldsymbol{r}}\right) \mu\left(d \widetilde{\boldsymbol{\xi}}_{\boldsymbol{r}}\right) \mu\left(d \widetilde{\boldsymbol{\eta}}_{\boldsymbol{p}-\boldsymbol{r}}\right)\right. \\
& \left.\times|\mathcal{F} \mathfrak{f}|^{2}\left(\boldsymbol{v}_{\boldsymbol{r}}, \widetilde{\boldsymbol{t}}_{\boldsymbol{p}-\boldsymbol{r}}, \boldsymbol{\eta}_{\boldsymbol{p}-\boldsymbol{r}}, \widetilde{\boldsymbol{\xi}}_{\boldsymbol{r}}\right)|\mathcal{F} \mathfrak{f}|^{2}\left(\widetilde{\boldsymbol{s}}_{\boldsymbol{r}}, \boldsymbol{w}_{\boldsymbol{p}-\boldsymbol{r}}, \widetilde{\boldsymbol{\eta}}_{\boldsymbol{p}-\boldsymbol{r}}, \boldsymbol{\xi}_{\boldsymbol{r}}\right) \ell_{R}(\widetilde{a}+b) \ell_{R}(a+\widetilde{b})\right]^{1 / 2} \\
& =: 4 U_{1, R, \delta}^{1 / 2} U_{2, R, \delta}^{1 / 2} .
\end{aligned}
$$

In what follows, we are going to prove separately in case (H1) and in case (H2) that (4.6) holds.

Proof of (4.6) under (H1): From (4.7), using Cauchy-Schwarz inequality again for the integration in time, we have

$$
\mathcal{Y}_{2, R, \delta} \leq 4 \sqrt{\mathfrak{X}_{1, R, \delta} \mathfrak{X}_{2, R, \delta}},
$$

where, for $k=1,2$,

$$
\begin{aligned}
& \mathfrak{X}_{k, R, \delta} \\
& :=\int_{[0, t]^{4 p}} d \ell^{4 p}\left(\prod_{i=1}^{r} \gamma_{0}\left(s_{i}-\widetilde{s}_{i}\right) \gamma_{0}\left(v_{i}-\widetilde{v}_{i}\right)\right)\left(\prod_{j=1}^{p-r} \gamma_{0}\left(t_{j}-\widetilde{t}_{j}\right) \gamma_{0}\left(w_{j}-\widetilde{w}_{j}\right)\right) U_{k, R, \delta .}
\end{aligned}
$$

One can show by the same arguments as before that, for all $R>1$ and $\delta>0$,

$$
\mathfrak{X}_{1, R, \delta} \leq \Gamma_{t}^{2 p} V_{12, R}^{2} \leq C .
$$

Now we write

$$
\begin{aligned}
\mathfrak{X}_{2, R, \delta} \leq & \Gamma_{t}^{2 p} \int_{[0, t]^{2 p}} d \widetilde{\boldsymbol{s}}_{\boldsymbol{r}} d \widetilde{\boldsymbol{t}}_{\boldsymbol{p}-\boldsymbol{r}} d \boldsymbol{v}_{\boldsymbol{r}} d \boldsymbol{w}_{\boldsymbol{p}-\boldsymbol{r}} \int_{\{|a+b|<\delta\} \times \mathbb{R}^{p}} \mu\left(d \boldsymbol{\xi}_{\boldsymbol{r}}\right) \mu\left(d \boldsymbol{\eta}_{\boldsymbol{p}-\boldsymbol{r}}\right) \mu\left(d \widetilde{\boldsymbol{\xi}}_{\boldsymbol{r}}\right) \mu\left(d \widetilde{\boldsymbol{\eta}}_{\boldsymbol{p}-\boldsymbol{r}}\right) \\
& \times|\mathcal{F} \mathfrak{f}|^{2}\left(\boldsymbol{v}_{\boldsymbol{r}}, \widetilde{\boldsymbol{t}}_{\boldsymbol{p}-\boldsymbol{r}}, \boldsymbol{\eta}_{\boldsymbol{p}-\boldsymbol{r}}, \widetilde{\boldsymbol{\xi}}_{\boldsymbol{r}}\right)|\mathcal{F} \mathfrak{f}|^{2}\left(\widetilde{\boldsymbol{s}}_{\boldsymbol{r}}, \boldsymbol{w}_{\boldsymbol{p}-\boldsymbol{r}}, \widetilde{\boldsymbol{\eta}}_{\boldsymbol{p}-\boldsymbol{r}}, \boldsymbol{\xi}_{\boldsymbol{r}}\right) \ell_{R}(\widetilde{a}+b) \ell_{R}(a+\widetilde{b}) \\
= & \Gamma_{t}^{2 p} \int_{\mathbb{R}^{2 p}} \mu\left(d \boldsymbol{\xi}_{\boldsymbol{p}}\right) \mu\left(d \widetilde{\boldsymbol{\xi}}_{\boldsymbol{p}}\right) \mathbf{1}_{\left\{\left|\xi_{1}+\cdots+\xi_{r}+\widetilde{\xi}_{r+1}+\cdots+\widetilde{\xi}_{p}\right|<\delta\right\}} \ell_{R}\left(\tau\left(\boldsymbol{\xi}_{\boldsymbol{p}}\right)\right) \ell_{R}\left(\tau\left(\widetilde{\boldsymbol{\xi}}_{\boldsymbol{p}}\right)\right) \\
& \times\left(\int_{[0, t]^{p}} d \boldsymbol{s}_{\boldsymbol{p}}|\mathcal{F} \mathfrak{f}|^{2}\left(\boldsymbol{s}_{\boldsymbol{p}}, \widetilde{\boldsymbol{\xi}}_{\boldsymbol{p}}\right)\right)\left(\int_{[0, t]^{p}} d \boldsymbol{t}_{\boldsymbol{p}}|\mathcal{F} \mathfrak{f}|^{2}\left(\boldsymbol{t}_{\boldsymbol{p}}, \boldsymbol{\xi}_{\boldsymbol{p}}\right)\right) .
\end{aligned}
$$


Using (4.1) and a change of variables in time, we can rewrite the last expression as follows, with $\eta_{0}=\widetilde{\eta}_{0}=0$,

$$
\begin{aligned}
& \mathfrak{X}_{2, R, \delta} \leq \frac{\Gamma_{t}^{2 p}}{(p !)^{4}} \int_{\mathbb{R}^{2 p}} \mu\left(d \boldsymbol{\xi}_{\boldsymbol{p}}\right) \mu\left(d \tilde{\boldsymbol{\xi}}_{\boldsymbol{p}}\right) \mathbf{1}_{\left\{\left|\xi_{1}+\cdots+\xi_{r}+\widetilde{\xi}_{r+1}+\cdots+\widetilde{\xi}_{p}\right|<\delta\right\}} \ell_{R}\left(\tau\left(\boldsymbol{\xi}_{\boldsymbol{p}}\right)\right) \ell_{R}\left(\tau\left(\widetilde{\boldsymbol{\xi}}_{\boldsymbol{p}}\right)\right) \\
& \times \int_{[0, t]^{2 p}} d \boldsymbol{s}_{\boldsymbol{p}} d \boldsymbol{t}_{\boldsymbol{p}} \exp \left(-\operatorname{Var} \sum_{j=1}^{p} B_{s_{j}} \widetilde{\xi}_{j}\right) \exp \left(-\operatorname{Var} \sum_{j=1}^{p} B_{t_{j}} \xi_{j}\right) \\
&=\frac{\Gamma_{t}^{2 p}}{(p !)^{2}} \int_{\mathbb{R}^{2 p}} d \boldsymbol{\eta}_{\boldsymbol{p}} d \widetilde{\boldsymbol{\eta}}_{\boldsymbol{p}}\left(\prod_{j=1}^{p} \varphi\left(\eta_{j}-\eta_{j-1}\right) \varphi\left(\widetilde{\eta}_{j}-\widetilde{\eta}_{j-1}\right)\right) \mathbf{1}_{\left\{\left|\eta_{r}+\widetilde{\eta}_{p}-\widetilde{\eta}_{r}\right|<\delta\right\}} \ell_{R}\left(\eta_{p}\right) \ell_{R}\left(\widetilde{\eta}_{p}\right) \\
& \quad \times \int_{\operatorname{SIM}_{p}(t)^{2}} d \boldsymbol{w}_{\boldsymbol{p}} d \widetilde{\boldsymbol{w}}_{\boldsymbol{p}} \prod_{j=1}^{p} e^{-w_{j} \eta_{j}^{2}} e^{-\widetilde{w}_{j} \widetilde{\eta}_{j}^{2}} \\
& \leq \int_{G(\delta)} d \boldsymbol{\eta}_{\boldsymbol{p}} d \widetilde{\boldsymbol{\eta}}_{\boldsymbol{p}} \Phi\left(\boldsymbol{\eta}_{\boldsymbol{p}}, \widetilde{\boldsymbol{\eta}}_{\boldsymbol{p}}\right),
\end{aligned}
$$

where $G(\delta)=\left\{\left(\boldsymbol{\eta}_{\boldsymbol{p}}, \widetilde{\boldsymbol{\eta}}_{\boldsymbol{p}}\right) \in \mathbb{R}^{2 p}:\left|\eta_{r}+\widetilde{\eta}_{p}-\widetilde{\eta}_{r}\right|<\delta\right\}$ and

$$
\begin{aligned}
\Phi\left(\boldsymbol{\eta}_{\boldsymbol{p}}, \widetilde{\boldsymbol{\eta}}_{\boldsymbol{p}}\right):= & \frac{\Gamma_{t}^{2 p} \kappa_{0}^{2(p-1)}}{(p !)^{2}} \sum_{\boldsymbol{\beta}, \widetilde{\boldsymbol{\beta}} \in \mathcal{A}_{p}}\left(\prod_{j=1}^{p} \varphi\left(\eta_{j}\right)^{\beta_{j}} \varphi\left(\widetilde{\eta}_{j}\right)^{\widetilde{\beta}_{j}}\right) \ell_{R}\left(\eta_{p}\right) \ell_{R}\left(\widetilde{\eta}_{p}\right) \\
& \times \int_{\operatorname{SIM}_{p}(t)^{2}} d \boldsymbol{w}_{\boldsymbol{p}} d \widetilde{\boldsymbol{w}}_{\boldsymbol{p}} \prod_{j=1}^{p} e^{-w_{j} \eta_{j}^{2}} e^{-\widetilde{\boldsymbol{w}}_{j} \widetilde{\eta}_{j}^{2}}
\end{aligned}
$$

Then, we decompose the set $G(\delta)$ as follows

$$
G(\delta) \subset\left\{\left|\eta_{r}-\widetilde{\eta}_{r}\right|<2 \delta\right\} \cup\left\{\left|\widetilde{\eta}_{p}\right| \geq \delta\right\} .
$$

From the estimation (4.4) for the term $V_{11, R, \delta}$, it follows that, for any $\delta>0$,

$$
\int_{\left\{\left|\widetilde{\eta}_{p}\right| \geq \delta\right\}} d \boldsymbol{\eta}_{\boldsymbol{p}} d \widetilde{\boldsymbol{\eta}}_{\boldsymbol{p}} \Phi\left(\boldsymbol{\eta}_{\boldsymbol{p}}, \widetilde{\boldsymbol{\eta}}_{\boldsymbol{p}}\right) \stackrel{R \rightarrow \infty}{\longrightarrow} 0 .
$$

On the other hand, from the proofs of the estimates (3.7) and (3.8) in Lemma 3.1, we obtain

$$
\begin{aligned}
\int_{\left\{\left|\eta_{r}-\widetilde{\eta}_{r}\right|<2 \delta\right\}} d \boldsymbol{\eta}_{\boldsymbol{p}} d \widetilde{\boldsymbol{\eta}}_{\boldsymbol{p}} \Phi\left(\boldsymbol{\eta}_{\boldsymbol{p}}, \widetilde{\boldsymbol{\eta}}_{\boldsymbol{p}}\right) \leq & C \sup _{\beta, \widetilde{\beta} \in\{0,1,2\}} \int_{\mathbb{R}^{2}} \int_{[0, t]^{2}} d \eta_{r} d \widetilde{\eta}_{r} d w_{r} d \widetilde{w}_{r} \varphi\left(\eta_{r}\right)^{\beta} e^{-w_{r} \eta_{r}^{2}} \\
& \times \varphi(\widetilde{\eta})^{\widetilde{\beta}} e^{-\widetilde{w}_{r} \widetilde{\eta}_{r}^{2}} \mathbf{1}_{\left\{\left|\eta_{r}-\widetilde{\eta}_{r}\right|<2 \delta\right\} .}
\end{aligned}
$$

Note also that for any $\beta, \widetilde{\beta} \in\{0,1,2\}$,

$$
\int_{\mathbb{R}^{2}} \int_{[0, t]^{2}} d \eta_{r} d \widetilde{\eta}_{r} d w_{r} d \widetilde{w}_{r} \varphi\left(\eta_{r}\right)^{\beta} e^{-w_{r} \eta_{r}^{2}} \varphi\left(\widetilde{\eta}_{r}\right)^{\widetilde{\beta}} e^{-\widetilde{w}_{r} \widetilde{\eta}_{r}^{2}} \leq C .
$$

Since $\mathbf{1}_{\left\{\left|\eta_{r}-\widetilde{\eta}_{r}\right|<2 \delta\right\}} \rightarrow 0$ as $\delta \rightarrow 0$, we deduce

$$
\lim _{\delta \rightarrow 0} \limsup _{R \rightarrow \infty} \int_{\left\{\left|\eta_{r}-\widetilde{\eta}_{r}\right|<2 \delta\right\}} d \boldsymbol{\eta}_{\boldsymbol{p}} d \widetilde{\boldsymbol{\eta}}_{\boldsymbol{p}} \Phi\left(\boldsymbol{\eta}_{\boldsymbol{p}}, \widetilde{\boldsymbol{\eta}}_{\boldsymbol{p}}\right)=0 .
$$

Thus, (4.8) and (4.9) allow us to complete the proof of (4.6) in case (H1). 
Proof of (4.6) under (H2): From (4.7), the embedding result (2.4) and CauchySchwarz inequality, it follows that

$$
\mathcal{Y}_{2, R, \delta} \leq C\left\{\int_{[0, t]^{2 p}} d \ell^{2 p} U_{1, R, \delta}^{\frac{1}{2 H_{0}}}\right\}^{H_{0}}\left\{\int_{[0, t]^{2 p}} d \ell^{2 p} U_{2, R, \delta}^{\frac{1}{2 H_{0}}}\right\}^{H_{0}}=: C \mathfrak{Z}_{1, R, \delta}^{H_{0}} \mathfrak{Z}_{2, R, \delta}^{H_{0}},
$$

where

$$
\begin{aligned}
\mathfrak{Z}_{1, R, \delta}= & \int_{[0, t]^{2 p}} d \boldsymbol{s}_{\boldsymbol{p}} d \boldsymbol{t}_{\boldsymbol{p}}\left(\int_{\mathbb{R}^{p}} \mu\left(d \boldsymbol{\xi}_{\boldsymbol{p}}\right) \ell_{R}\left(\tau\left(\boldsymbol{\xi}_{\boldsymbol{p}}\right)\right)|\mathcal{F} \mathfrak{f}|^{2}\left(\boldsymbol{s}_{\boldsymbol{p}}, \boldsymbol{\xi}_{\boldsymbol{p}}\right)\right. \\
& \left.\times \int_{\left\{\left|\tau\left(\boldsymbol{\xi}_{\boldsymbol{p}}\right)\right|<\delta\right\}} \mu\left(d \boldsymbol{\xi}_{\boldsymbol{p}}\right) \ell_{R}\left(\tau\left(\boldsymbol{\xi}_{\boldsymbol{p}}\right)\right)|\mathcal{F} \mathfrak{f}|^{2}\left(\boldsymbol{t}_{\boldsymbol{p}}, \boldsymbol{\xi}_{\boldsymbol{p}}\right)\right)^{\frac{1}{2 H_{0}}} \\
\mathfrak{Z}_{2, R, \delta}= & \int_{[0, t]^{2 p}} d \boldsymbol{s}_{\boldsymbol{p}} d \boldsymbol{t}_{\boldsymbol{p}}\left(\int_{\{|a+b|<\delta\} \times \mathbb{R}^{p}} \mu\left(d \boldsymbol{\xi}_{\boldsymbol{r}}\right) \mu\left(d \boldsymbol{\eta}_{\boldsymbol{p}-\boldsymbol{r}}\right) \mu\left(d \widetilde{\boldsymbol{\xi}}_{\boldsymbol{r}}\right) \mu\left(d \widetilde{\boldsymbol{\eta}}_{\boldsymbol{p}-\boldsymbol{r}}\right)\right. \\
& \left.\times|\mathcal{F} \mathfrak{f}|^{2}\left(\boldsymbol{s}_{\boldsymbol{p}}, \boldsymbol{\eta}_{\boldsymbol{p}-\boldsymbol{r}}, \widetilde{\boldsymbol{\xi}}_{\boldsymbol{r}}\right)|\mathcal{F} \mathfrak{f}|^{2}\left(\widetilde{\boldsymbol{t}}_{\boldsymbol{p}}, \widetilde{\boldsymbol{\eta}}_{\boldsymbol{p}-\boldsymbol{r}}, \boldsymbol{\xi}_{\boldsymbol{r}}\right) \ell_{R}(\widetilde{a}+b) \ell_{R}(a+\widetilde{b})\right)^{\frac{1}{2 H_{0}}} .
\end{aligned}
$$

For the term $\mathfrak{Z}_{1, R, \delta}$, we deduce from (4.5) that

$$
\mathfrak{Z}_{1, R, \delta} \leq\left(\int_{[0, t]^{p}} d \boldsymbol{s}_{\boldsymbol{p}}\left(\int_{\mathbb{R}^{p}} \mu\left(d \boldsymbol{\xi}_{\boldsymbol{p}}\right) \ell_{R}\left(\tau\left(\boldsymbol{\xi}_{\boldsymbol{p}}\right)\right)|\mathcal{F} \mathfrak{f}|^{2}\left(\boldsymbol{s}_{\boldsymbol{p}}, \boldsymbol{\xi}_{\boldsymbol{p}}\right)\right)^{\frac{1}{2 H_{0}}}\right)^{2}=T_{2, R}^{2} \leq C
$$

for all $R>1$ and $\delta>0$, which shows the uniform boundedness of $\mathfrak{Z}_{1, R, \delta}$ over $R>1$ and $\delta>0$.

Now let us consider the term $\mathfrak{Z}_{2, R, \delta}$. Similar to the analysis on the term $\mathcal{Y}_{1, R, \delta}$, one can get, with $\eta_{0}=\widetilde{\eta}_{0}=0$,

$$
\begin{aligned}
& \mathfrak{Z}_{2, R, \delta}=(p !)^{-\frac{2\left(1-H_{0}\right)}{H_{0}}} \int_{\operatorname{SIM}_{p}(t)^{2}} d \boldsymbol{w}_{\boldsymbol{p}} d \widetilde{\boldsymbol{w}}_{\boldsymbol{p}}\left(\int_{\mathbb{R}^{2 p}} d \boldsymbol{\eta}_{\boldsymbol{p}} d \widetilde{\boldsymbol{\eta}}_{\boldsymbol{p}} \mathbf{1}_{\left\{\left|\eta_{r}+\widetilde{\boldsymbol{\eta}}_{p}-\widetilde{\boldsymbol{\eta}}_{r}\right|<\delta\right\}} \ell_{R}\left(\eta_{p}\right) \ell_{R}\left(\widetilde{\eta}_{p}\right)\right. \\
& \left.\times \prod_{j=1}^{p}\left|\eta_{j}-\eta_{j-1}\right|^{1-2 H_{1}} e^{-w_{j} \eta_{j}^{2}} \prod_{j=1}^{p}\left|\widetilde{\eta}_{j}-\widetilde{\eta}_{j-1}\right|^{1-2 H_{1}} e^{-\widetilde{w}_{j} \widetilde{\eta}_{j}^{2}}\right)^{\frac{1}{2 H_{0}}} \\
& =(p !)^{-\frac{2\left(1-H_{0}\right)}{H_{0}}} \int_{\operatorname{SIM}_{p}(t)^{2}} d \boldsymbol{w}_{\boldsymbol{p}} d \widetilde{\boldsymbol{w}}_{\boldsymbol{p}}\left(\int_{\mathbb{R}^{2 p}} d \boldsymbol{\eta}_{\boldsymbol{p}} d \widetilde{\boldsymbol{\eta}}_{\boldsymbol{p}} \mathbf{1}_{\left\{\left|\eta_{r}+\widetilde{\eta}_{p}-\widetilde{\boldsymbol{\eta}}_{r}\right|<\delta\right\}} \ell_{R}\left(\eta_{p}\right) \ell_{R}\left(\widetilde{\eta}_{p}\right)\right. \\
& \left.\prod_{j=1}^{p}\left|\eta_{j}-\eta_{j-1}\right|^{1-2 H_{1}} e^{-w_{j} \eta_{j}^{2}}\left(\mathbf{1}_{\left\{\left|\widetilde{\eta}_{p}\right|<\delta\right\}}+\mathbf{1}_{\left\{\left|\widetilde{\eta}_{p}\right| \geq \delta\right\}}\right) \prod_{j=1}^{p}\left|\widetilde{\eta}_{j}-\widetilde{\eta}_{j-1}\right|^{1-2 H_{1}} e^{-\widetilde{w}_{j} \widetilde{\eta}_{j}^{2}}\right)^{\frac{1}{2 H_{0}}} \\
& =: \mathfrak{Z}_{21, R, \delta}+\mathfrak{Z}_{22, R, \delta},
\end{aligned}
$$

where

$$
\begin{aligned}
\mathfrak{Z}_{21, R, \delta} & =(p !)^{-\frac{2\left(1-H_{0}\right)}{H_{0}}} \int_{\operatorname{SIM}_{p}(t)^{2}} d \boldsymbol{w}_{\boldsymbol{p}} d \widetilde{\boldsymbol{w}}_{\boldsymbol{p}}\left(\int_{\mathbb{R}^{2 p}} d \boldsymbol{\eta}_{\boldsymbol{p}} d \widetilde{\boldsymbol{\eta}}_{\boldsymbol{p}} \mathbf{1}_{\left\{\left|\eta_{r}+\widetilde{\eta}_{p}-\widetilde{\eta}_{r}\right|<\delta\right\}} \mathbf{1}_{\left\{\left|\widetilde{\eta}_{p}\right|<\delta\right\}}\right. \\
& \left.\times \ell_{R}\left(\eta_{p}\right) \ell_{R}\left(\widetilde{\eta}_{p}\right) \prod_{j=1}^{p}\left|\eta_{j}-\eta_{j-1}\right|^{1-2 H_{1}} e^{-w_{j} \eta_{j}^{2}} \prod_{j=1}^{p}\left|\widetilde{\eta}_{j}-\widetilde{\eta}_{j-1}\right|^{1-2 H_{1}} e^{-\widetilde{w}_{j} \widetilde{\eta}_{j}^{2}}\right)^{\frac{1}{2 H_{0}}}
\end{aligned}
$$


and

$$
\begin{aligned}
\mathfrak{Z}_{22, R, \delta} & =(p !)^{-\frac{2\left(1-H_{0}\right)}{H_{0}}} \int_{\operatorname{SIM}_{p}(t)^{2}} d \boldsymbol{w}_{\boldsymbol{p}} d \widetilde{\boldsymbol{w}}_{\boldsymbol{p}}\left(\int_{\mathbb{R}^{2 p}} d \boldsymbol{\eta}_{\boldsymbol{p}} d \widetilde{\boldsymbol{\eta}}_{\boldsymbol{p}} \mathbf{1}_{\left\{\left|\eta_{r}+\widetilde{\eta}_{p}-\widetilde{\eta}_{r}\right|<\delta\right\}} \mathbf{1}_{\left\{\left|\widetilde{\eta}_{p}\right| \geq \delta\right\}}\right. \\
& \left.\times \ell_{R}\left(\eta_{p}\right) \ell_{R}\left(\widetilde{\eta}_{p}\right) \prod_{j=1}^{p}\left|\eta_{j}-\eta_{j-1}\right|^{1-2 H_{1}} e^{-w_{j} \eta_{j}^{2}} \prod_{j=1}^{p}\left|\widetilde{\eta}_{j}-\widetilde{\eta}_{j-1}\right|^{1-2 H_{1}} e^{-\widetilde{w}_{j} \widetilde{\eta}_{j}^{2}}\right)^{\frac{1}{2 H_{0}}} .
\end{aligned}
$$

For $\mathfrak{Z}_{21, R, \delta}$, we have

$$
\begin{aligned}
& \mathfrak{Z}_{21, R, \delta} \leq C \sum_{\boldsymbol{\beta}, \widetilde{\boldsymbol{\beta}} \in \mathcal{A}_{p}} \int_{\operatorname{SIM}_{p}(t)^{2}} d \boldsymbol{w}_{\boldsymbol{p}} d \widetilde{\boldsymbol{w}}_{\boldsymbol{p}}\left(\int_{\mathbb{R}^{2 p}} d \boldsymbol{\eta}_{\boldsymbol{p}} d \widetilde{\boldsymbol{\eta}}_{\boldsymbol{p}} \mathbf{1}_{\left\{\left|\eta_{r}-\widetilde{\eta}_{r}\right|<2 \delta\right\}} \ell_{R}\left(\eta_{p}\right) \ell_{R}\left(\widetilde{\eta}_{p}\right)\right. \\
& \left.\times \prod_{j=1}^{p}\left|\eta_{j}\right|^{\beta_{j}\left(1-2 H_{1}\right)} e^{-w_{j} \eta_{j}^{2}} \prod_{j=1}^{p}\left|\widetilde{\eta}_{j}\right|^{\widetilde{\beta}_{j}\left(1-2 H_{1}\right)} e^{-\widetilde{w}_{j} \widetilde{\eta}_{j}^{2}}\right)^{\frac{1}{2 H_{0}}} \\
& \leq C \sum_{\boldsymbol{\beta}, \widetilde{\boldsymbol{\beta}} \in \mathcal{A}_{p}}\left(\int_{[0, t]} d w_{p}\left(\int_{\mathbb{R}} d \eta_{p} \ell_{R}\left(\eta_{p}\right)\left|\eta_{p}\right|^{\beta_{p}\left(1-2 H_{1}\right)} e^{-w_{p} \eta_{p}^{2}}\right)^{\frac{1}{2 H_{0}}}\right)^{2} \\
& \times\left(\int_{\operatorname{SIM}_{p-2}(t)} d \boldsymbol{w}_{\boldsymbol{p - 2}}\left(\int_{\mathbb{R}^{(p-2)}} d \boldsymbol{\eta}_{\boldsymbol{p - 2}} \prod_{j \in\{1, \ldots, p-1\}, j \neq r}\left|\eta_{j}\right|^{\beta_{j}\left(1-2 H_{1}\right)} e^{-w_{j} \eta_{j}^{2}}\right)^{\frac{1}{2 H_{0}}}\right)^{2} \\
& \int_{[0, t]^{2}} d w_{r} d \widetilde{w}_{r}\left(\int_{\mathbb{R}^{2}} d \eta_{r} d \widetilde{\eta}_{r} \mathbf{1}_{\left\{\left|\eta_{r}-\widetilde{\eta}_{r}\right|<2 \delta\right\}}\left|\eta_{r}\right|^{\beta_{r}\left(1-2 H_{1}\right)}\left|\widetilde{\eta}_{r}\right|^{\widetilde{\beta}_{r}\left(1-2 H_{1}\right)} e^{-w_{r} \eta_{r}^{2}-\widetilde{w}_{r} \widetilde{\eta}_{r}^{2}}\right)^{\frac{1}{2 H_{0}}} .
\end{aligned}
$$

Using some previous calculations, we obtain the following bound

$$
\begin{aligned}
& \sup _{R>1} \mathfrak{Z}_{21, R, \delta} \\
& \quad \leq C \int_{[0, t]^{2}} d v d s\left(\int_{\mathbb{R}^{2}} d x d y \mathbf{1}_{\{|x-y|<2 \delta\}}|x|^{\beta_{r}\left(1-2 H_{1}\right)}|y|^{\widetilde{\beta}_{r}\left(1-2 H_{1}\right)} e^{-v x^{2}-s y^{2}}\right)^{\frac{1}{2 H_{0}}}
\end{aligned}
$$

where $\beta_{r}, \widetilde{\beta}_{r} \in\{0,1,2\}$ and this proves, by the dominated convergence theorem, that

$$
\lim _{\delta \rightarrow 0} \limsup _{R>1} \mathfrak{Z}_{21, R, \delta}=0 .
$$

On the other hand, the term $\mathfrak{Z}_{22, R, \delta}$ can be treated as $T_{1, R, \delta}$ and we obtain

$$
\lim _{R \rightarrow \infty} \mathfrak{Z}_{22, R, \delta}=0, \text { for any fixed } \delta>0 .
$$

Hence, the proof of (4.6) under (H2) is completed and this ends the proof of Proposition 4.1.

\section{Proof of tightness}

In this section, we will give the proof of the tightness by following the strategy proposed in Nualart and Zheng (2020a, Section 3.3). 
Proposition 5.1. For any fixed $T>0$, any $0<s<t \leq T \leq R$ and any integer $k \geq 2$

$$
\frac{1}{\sqrt{R}}\left\|A_{t}(R)-A_{s}(R)\right\|_{k} \leq C|t-s|^{1 / 2},
$$

where $C=C_{T, k}$ is a constant that depends on $T$ and $k$.

Proof: From the definition of mild solution (see Definition 2.2), we can write

$$
u(t, x)=1+\int_{\mathbb{R}_{+} \times \mathbb{R}} G\left(t-s_{1}, x-y_{1}\right) \mathbf{1}_{[0, t)}\left(s_{1}\right) u\left(s_{1}, y_{1}\right) W\left(d s_{1}, d y_{1}\right) .
$$

Therefore, for $s<t$ we have the decomposition of $u(t, x)-u(s, x)$ into

$$
\begin{aligned}
\int_{\mathbb{R}_{+} \times \mathbb{R}} d_{1}\left(s, t, x ; s_{1}, y_{1}\right) u\left(s_{1}, y_{1}\right) & W\left(d s_{1}, d y_{1}\right) \\
+ & \int_{\mathbb{R}_{+} \times \mathbb{R}} d_{2}\left(s, t, x ; s_{1}, y_{1}\right) u\left(s_{1}, y_{1}\right) W\left(d s_{1}, d y_{1}\right),
\end{aligned}
$$

with $d_{1}\left(s, t, x ; s_{1}, y_{1}\right)=\mathbf{1}_{[0, s)}\left(s_{1}\right)\left[G\left(t-s_{1}, x-y_{1}\right)-G\left(s-s_{1}, x-y_{1}\right)\right]$ and

$$
d_{2}\left(s, t, x ; s_{1}, y_{1}\right)=\mathbf{1}_{[s, t)}\left(s_{1}\right) G\left(t-s_{1}, x-y_{1}\right) .
$$

Now we express $A_{t}(R)-A_{s}(R)$ as a sum of two chaos expansions that correspond to $d_{1}$ and $d_{2}$ :

$$
A_{t}(R)-A_{s}(R)=\sum_{p=1}^{\infty} J_{1, p, R}+\sum_{q=1}^{\infty} J_{2, q, R},
$$

where $J_{i, p, R}=\int_{B_{R}} I_{p}^{W}\left(\mathfrak{g}_{i, p, x}\right) d x$ for $i \in\{1,2\}$ and

$$
\begin{aligned}
\mathfrak{g}_{1, p, x}\left(\boldsymbol{s}_{\boldsymbol{p}}, \boldsymbol{y}_{\boldsymbol{p}}\right)= & \frac{1}{p !} \sum_{\sigma \in \mathfrak{S}_{p}} \mathbf{1}_{\Delta_{p}(s)}\left(\boldsymbol{s}_{\boldsymbol{p}}^{\boldsymbol{\sigma}}\right) d_{1}\left(s, t, x ; s_{\sigma(1)}, y_{\sigma(1)}\right) \\
& \times \prod_{j=1}^{p-1} G\left(s_{\sigma(j)}-s_{\sigma(j+1)}, y_{\sigma(j)}-y_{\sigma(j+1)}\right), \\
\mathfrak{g}_{2, p, x}\left(\boldsymbol{s}_{\boldsymbol{p}}, \boldsymbol{y}_{\boldsymbol{p}}\right)= & \frac{1}{p !} \sum_{\sigma \in \mathfrak{S}_{p}} \mathbf{1}_{\Delta_{p}(s, t)}\left(\boldsymbol{s}_{\boldsymbol{p}}^{\boldsymbol{\sigma}}\right) G\left(t-s_{\sigma(1)}, x-y_{\sigma(1)}\right) \\
& \times \prod_{j=1}^{p-1} G\left(s_{\sigma(j)}-s_{\sigma(j+1)}, y_{\sigma(j)}-y_{\sigma(j+1)}\right),
\end{aligned}
$$

with $\Delta_{p}(s, t)=\left\{t>s_{1}>\cdots>s_{p}>s\right\}$. Finally, we apply (2.1) to get

$$
\begin{aligned}
\frac{1}{\sqrt{R}}\left\|A_{t}(R)-A_{s}(R)\right\|_{k} & \leq \frac{1}{\sqrt{R}} \sum_{p=1}^{\infty}\left(\left\|J_{1, p, R}\right\|_{k}+\left\|J_{2, p, R}\right\|_{k}\right) \\
& \leq \frac{1}{\sqrt{R}} \sum_{p=1}^{\infty}(k-1)^{p / 2}\left(\left\|J_{1, p, R}\right\|_{2}+\left\|J_{2, p, R}\right\|_{2}\right) .
\end{aligned}
$$

Now, let us estimate $\left\|J_{i, p, R}\right\|_{2}$ for $i \in\{1,2\}$ and $p \in\{1,2, \ldots\}$. 
Case $i=2, p \geq 2$ : Following the arguments in Nualart and Zheng (2020a, Section 3.3), we write

$$
\begin{aligned}
\left\|J_{2, p, R}\right\|_{2}^{2} & =p ! \int_{-R}^{R} \int_{-R}^{R} d x d y\left\langle\mathfrak{g}_{2, p, x}, \mathfrak{g}_{2, p, y}\right\rangle_{\mathfrak{H} \otimes p} \\
& =\frac{1}{p !} \int_{-R}^{R} \int_{-R}^{R} d x d y \mathbb{E}\left[\left(\mathcal{I}_{t-s, t-s}^{1,2}(x-y)\right)^{p}\right] \leq \frac{2 R}{p !} \int_{\mathbb{R}} d z \mathbb{E}\left[\left|\mathcal{I}_{t-s, t-s}^{1,2}(z)\right|^{p}\right] .
\end{aligned}
$$

From (3.23) and (3.24), in view of the factor $t$ in the right-hand side of (3.23) and the factor $t^{4 H_{0} \mathfrak{h}+\frac{1}{2}}$ in the right-hand side of (3.24), with $4 H_{0} \mathfrak{h}+\frac{1}{2}>1$, it follows easily that

$$
\sum_{p=2}^{\infty}\left(\frac{1}{p !} \int_{\mathbb{R}} d z \mathbb{E}\left[\left|\mathcal{I}_{t-s, t-s}^{1,2}(z)\right|^{p}\right]\right)^{1 / 2}(k-1)^{p / 2} \leq C(t-s)^{1 / 2}
$$

Hence, $\frac{1}{\sqrt{R}} \sum_{p=2}^{\infty}\left\|J_{2, p, R}\right\|_{k} \leq C(t-s)^{1 / 2}$.

Case $i=2, p=1$ : We have $\mathfrak{g}_{2,1, x}\left(s_{1}, y_{1}\right)=\mathbf{1}_{[s, t]}\left(s_{1}\right) G\left(t-s_{1}, x-y_{1}\right)$, so it follows from (3.15) that

$$
\begin{aligned}
& \frac{1}{4 \pi R}\left\|J_{2,1, R}\right\|_{2}^{2} \\
& =\frac{1}{4 \pi R} \int_{s}^{t} \int_{s}^{t} d r d v \gamma_{0}(r-v) \int_{\mathbb{R}} d \xi \int_{-R}^{R} \int_{-R}^{R} d x d y e^{-\mathbf{i}(x-y) \xi} \varphi(\xi) e^{-\frac{t-r+t-v}{2}} \xi^{2} \\
& =\int_{s}^{t} \int_{s}^{t} d r d v \gamma_{0}(r-v) \int_{\mathbb{R}} d \xi \ell_{R}(\xi) \varphi(\xi) e^{-\frac{t-r+t-v}{2} \xi^{2}} .
\end{aligned}
$$

Then, from (3.16) we obtain

$$
\frac{1}{4 \pi R}\left\|J_{2,1, R}\right\|_{2}^{2} \leq(t-s) \Gamma_{T} \int_{\mathbb{R}} d \xi \ell_{R}(\xi) \varphi(\xi) \leq C(t-s) .
$$

As a consequence,

$$
\frac{1}{\sqrt{R}}\left\|J_{2,1, R}\right\|_{2} \leq C \sqrt{t-s} \text { so that } \frac{1}{\sqrt{R}}\left\|J_{2,1, R}\right\|_{k} \leq C \sqrt{t-s} .
$$

Therefore, we have proved

$$
\frac{1}{\sqrt{R}} \sum_{p=1}^{\infty}\left\|J_{2, p, R}\right\|_{k} \leq C(t-s)^{1 / 2}
$$

Next, we shall prove

$$
\frac{1}{\sqrt{R}} \sum_{p=1}^{\infty}\left\|J_{1, p, R}\right\|_{k} \leq C(t-s)^{1 / 2}
$$


Case $i=1, p=1$ : We have $\mathfrak{g}_{1,1, x}\left(s_{1}, y_{1}\right)=\mathbf{1}_{\left\{s_{1}<s\right\}}\left[G\left(t-s_{1}, x-y_{1}\right)-G\left(s-s_{1}, x-\right.\right.$ $\left.y_{1}\right)$ ] and then,

$$
\begin{aligned}
& \frac{1}{4 \pi R}\left\|J_{1,1, R}\right\|_{2}^{2}=\int_{0}^{s} \int_{0}^{s} d r d v \gamma_{0}(r-v) \int_{\mathbb{R}} d \xi \varphi(\xi) \ell_{R}(\xi)\left(e^{-\frac{t-r}{2} \xi^{2}}-e^{-\frac{s-r}{2} \xi^{2}}\right) \\
& \times\left(e^{-\frac{t-v}{2} \xi^{2}}-e^{-\frac{s-v}{2} \xi^{2}}\right) \\
& \leq \int_{0}^{s} \int_{0}^{s} d r d v \gamma_{0}(r-v) \int_{\mathbb{R}} d \xi \varphi(\xi) \ell_{R}(\xi) e^{-\frac{s-r}{2} \xi^{2}}\left(1-e^{-\frac{t-s}{2} \xi^{2}}\right) \\
& \leq \frac{t-s}{2} \int_{0}^{s} \int_{0}^{s} d r d v \gamma_{0}(r-v) \int_{\mathbb{R}} d \xi \varphi(\xi) \ell_{R}(\xi) e^{-\frac{s-r}{2} \xi^{2}} \xi^{2} \quad \text { as } 1-e^{-x} \leq x, \forall x \geq 0 \\
& \leq \frac{t-s}{2} \Gamma_{s} \int_{\mathbb{R}} d \xi \varphi(\xi) \ell_{R}(\xi) \xi^{2} \int_{0}^{s} d r e^{-\frac{s-r}{2} \xi^{2}} \\
& =(t-s) \Gamma_{s} \int_{\mathbb{R}} d \xi \varphi(\xi) \ell_{R}(\xi) \xi^{2} \frac{1-e^{-\frac{s \xi^{2}}{2}}}{\xi^{2}} \leq(t-s) \Gamma_{T} \int_{\mathbb{R}} d \xi \varphi(\xi) \ell_{R}(\xi) \leq C(t-s),
\end{aligned}
$$

for $R \geq T$, in view of (3.16). This implies

$$
\frac{1}{\sqrt{R}}\left\|J_{1,1, R}\right\|_{2} \leq C \sqrt{t-s} \text { so that } \frac{1}{\sqrt{R}}\left\|J_{1,1, R}\right\|_{k} \leq C \sqrt{t-s} .
$$

Case $i=1, p \geq 2$ : Let us first compute the Fourier transform of $\mathfrak{g}_{1, p, x}$

$$
\begin{aligned}
& \left(\mathcal{F} \mathfrak{g}_{1, p, x}\right)\left(\boldsymbol{s}_{\boldsymbol{p}}, \boldsymbol{\xi}_{\boldsymbol{p}}\right)=\frac{1}{p !} \sum_{\sigma \in \mathfrak{S}_{p}} \mathbf{1}_{\Delta_{p}(s)}\left(\boldsymbol{s}_{\boldsymbol{p}}^{\boldsymbol{\sigma}}\right) \int_{\mathbb{R}^{p}} d \boldsymbol{y}_{\boldsymbol{p}} e^{-\mathbf{i} \boldsymbol{\xi}_{\boldsymbol{p}} \cdot \boldsymbol{y}_{\boldsymbol{p}}} \\
& \quad \times\left[G\left(t-s_{\sigma(1)}, x-y_{\sigma(1)}\right)-G\left(s-s_{\sigma(1)}, x-y_{\sigma(1)}\right)\right] \\
& \quad \times \prod_{j=1}^{p-1} G\left(s_{\sigma(j)}-s_{\sigma(j+1)}, y_{\sigma(j)}-y_{\sigma(j+1)}\right) \\
& =e^{-\mathbf{i} \tau\left(\boldsymbol{\xi}_{\boldsymbol{p}}\right) x} \frac{1}{p !} \mathbf{1}_{[0, s]}\left(\boldsymbol{s}_{\boldsymbol{p}}\right)\left(\mathbb{E}\left[e^{-\mathbf{i} \sum_{j=1}^{p}\left(B_{t}-B_{s_{j}}\right) \xi_{j}}\right]-\mathbb{E}\left[e^{-\mathbf{i} \sum_{j=1}^{p}\left(B_{s}-B_{s_{j}}\right) \xi_{j}}\right]\right) .
\end{aligned}
$$

Therefore,

$$
\begin{aligned}
& \frac{1}{4 \pi R}\left\|J_{1, p, R}\right\|_{2}^{2}=\frac{p !}{4 \pi R} \int_{-R}^{R} \int_{-R}^{R} d x d y\left\langle\mathfrak{g}_{1, p, x}, \mathfrak{g}_{1, p, y}\right\rangle_{\mathfrak{H} \otimes p} \\
= & \frac{p !}{4 \pi R} \int_{-R}^{R} \int_{-R}^{R} d x d y \int_{[0, s]^{2 p}} d \boldsymbol{s}_{\boldsymbol{p}} d \boldsymbol{r}_{\boldsymbol{p}} \prod_{i=1}^{p} \gamma_{0}\left(s_{i}-r_{i}\right) \int_{\mathbb{R}^{p}} \mu\left(d \boldsymbol{\xi}_{\boldsymbol{p}}\right)\left(\mathcal{F} \mathfrak{g}_{1, p, x}\right)\left(\boldsymbol{s}_{\boldsymbol{p}}, \boldsymbol{\xi}_{\boldsymbol{p}}\right) \\
& \times\left(\mathcal{F}_{\mathfrak{g}_{1, p, y}}\right)\left(\boldsymbol{r}_{\boldsymbol{p}},-\boldsymbol{\xi}_{\boldsymbol{p}}\right) \\
= & \frac{1}{p !} \int_{[0, s]^{2 p}} d \boldsymbol{s}_{\boldsymbol{p}} d \boldsymbol{r}_{\boldsymbol{p}}\left(\prod_{i=1}^{p} \gamma_{0}\left(s_{i}-r_{i}\right)\right) \int_{\mathbb{R}^{p}} \mu\left(d \boldsymbol{\xi}_{\boldsymbol{p}}\right) \ell_{R}\left(\tau\left(\boldsymbol{\xi}_{\boldsymbol{p}}\right)\right)\left(\mathbb{E}\left[e^{-\mathbf{i} \sum_{j=1}^{p}\left(B_{t}-B_{s_{j}}\right) \xi_{j}}\right]\right. \\
- & \left.\mathbb{E}\left[e^{-\mathbf{i} \sum_{j=1}^{p}\left(B_{s}-B_{s_{j}}\right) \xi_{j}}\right]\right)\left(\mathbb{E}\left[e^{-\mathbf{i} \sum_{j=1}^{p}\left(B_{t}-B_{r_{j}}\right) \xi_{j}}\right]-\mathbb{E}\left[e^{-\mathbf{i} \sum_{j=1}^{p}\left(B_{s}-B_{r_{j}}\right) \xi_{j}}\right]\right) .
\end{aligned}
$$

Let us consider separately case (H1) and case (H2). 
In case $(\mathbf{H 1} \mathbf{1})$, using the local integrability of $\gamma_{0}$ and the fact

$$
\mathbb{E}\left[e^{-\mathbf{i} \sum_{j=1}^{p}\left(B_{s}-B_{r_{j}}\right) \xi_{j}}\right]-\mathbb{E}\left[e^{-\mathbf{i} \sum_{j=1}^{p}\left(B_{t}-B_{r_{j}}\right) \xi_{j}}\right] \in[0,1],
$$

we can write

$$
\begin{aligned}
\frac{1}{4 \pi R}\left\|J_{1, p, R}\right\|_{2}^{2} \leq \frac{\Gamma_{s}^{p}}{p !} \int_{[0, s]^{p}} d \boldsymbol{s}_{\boldsymbol{p}} \int_{\mathbb{R}^{p}} \mu\left(d \boldsymbol{\xi}_{\boldsymbol{p}}\right) \ell_{R}\left(\tau\left(\boldsymbol{\xi}_{\boldsymbol{p}}\right)\right) \\
\\
\quad \times\left(\mathbb{E}\left[e^{-\mathbf{i} \sum_{j=1}^{p}\left(B_{s}-B_{s_{j}}\right) \xi_{j}}\right]-\mathbb{E}\left[e^{-\mathbf{i} \sum_{j=1}^{p}\left(B_{t}-B_{s_{j}}\right) \xi_{j}}\right]\right) .
\end{aligned}
$$

We have

$$
\begin{aligned}
0 & \leq \mathbb{E}\left[e^{-\mathbf{i} \sum_{j=1}^{p}\left(B_{s}-B_{s_{j}}\right) \xi_{j}}\right]-\mathbb{E}\left[e^{-\mathbf{i} \sum_{j=1}^{p}\left(B_{t}-B_{s_{j}}\right) \xi_{j}}\right] \\
& =\mathbb{E}\left[e^{-\mathbf{i} \sum_{j=1}^{p}\left(B_{s}-B_{s_{j}}\right) \xi_{j}}\left(1-e^{-\mathbf{i}\left(B_{t}-B_{s}\right) \tau\left(\boldsymbol{\xi}_{\boldsymbol{p}}\right)}\right)\right] \\
& =\mathbb{E}\left[e^{-\mathbf{i} \sum_{j=1}^{p}\left(B_{s}-B_{s_{j}}\right) \xi_{j}}\right]\left(1-e^{-\frac{1}{2}(t-s) \tau\left(\boldsymbol{\xi}_{\boldsymbol{p}}\right)^{2}}\right) \\
& \leq \frac{t-s}{2} \tau\left(\boldsymbol{\xi}_{\boldsymbol{p}}\right)^{2} \mathbb{E}\left[e^{-\mathbf{i} \sum_{j=1}^{p}\left(B_{s}-B_{s_{j}}\right) \xi_{j}}\right] .
\end{aligned}
$$

As a consequence, for all $R>T$, we can write

$$
\begin{aligned}
& \frac{1}{4 \pi R}\left\|J_{1, p, R}\right\|_{2}^{2} \\
& \leq \frac{(t-s) \Gamma_{s}^{p}}{2 p !} \int_{[0, s]^{p} \times \mathbb{R}^{p}} d \boldsymbol{s}_{\boldsymbol{p}} \mu\left(d \boldsymbol{\xi}_{\boldsymbol{p}}\right) \ell_{R}\left(\tau\left(\boldsymbol{\xi}_{\boldsymbol{p}}\right)\right) \tau\left(\boldsymbol{\xi}_{\boldsymbol{p}}\right)^{2} \mathbb{E}\left(e^{-\mathbf{i} \sum_{j=1}^{p}\left(B_{s}-B_{s_{j}}\right) \xi_{j}}\right) \\
& \leq \frac{(t-s) \Gamma_{T}^{p}}{2 p !} \int_{[0, s]^{p}} d \boldsymbol{s}_{\boldsymbol{p}} \int_{\mathbb{R}^{p}} \mu\left(d \boldsymbol{\xi}_{\boldsymbol{p}}\right) \frac{\sin ^{2}\left(R \tau\left(\boldsymbol{\xi}_{\boldsymbol{p}}\right)\right)}{\pi R} \mathbb{E}\left[e^{-\mathbf{i} \sum_{j=1}^{p}\left(B_{s}-B_{s_{j}}\right) \xi_{j}}\right] \\
& \leq \frac{(t-s) \Gamma_{T}^{p}}{2 \pi T p !} \int_{[0, s]^{p}} d \boldsymbol{s}_{\boldsymbol{p}} \int_{\mathbb{R}^{p}} \mu\left(d \boldsymbol{\xi}_{\boldsymbol{p}}\right) \mathbb{E}\left[e^{-\mathbf{i} \sum_{j=1}^{p} B_{s_{j}} \xi_{j}}\right]=\frac{(t-s) \Gamma_{T}^{p}}{2 \pi T p !} K_{1, p}(1, s) .
\end{aligned}
$$

Then, applying inequality (3.7), yields, for $R>T$,

$$
\begin{aligned}
& \frac{1}{\sqrt{R}} \sum_{p=2}^{\infty}\left\|J_{1, p, R}\right\|_{k} \leq \frac{1}{\sqrt{R}} \sum_{p=2}^{\infty}\left\|J_{1, p, R}\right\|_{2}(k-1)^{p / 2} \\
& \leq \sqrt{\frac{1}{T \kappa_{0}}}(t-s)^{1 / 2} \sum_{p=2}^{\infty} \sqrt{\left(8 \kappa_{0} C_{N}(k-1) \Gamma_{T}\right)^{p} \exp \left(\frac{s D_{N}}{2 C_{N}}\right)} \leq C(t-s)^{1 / 2},
\end{aligned}
$$

for some large $N$ such that $8 \kappa_{0} C_{N}^{\prime}(k-1) \Gamma_{T}<1$. This, together with the previous estimate for $p=1$, leads to (5.3) under the hypothesis (H1).

Consider now the case (H2). Let us continue with (5.4) and apply the embedding result (2.4) and Cauchy-Schwarz inequality to write

$$
\begin{aligned}
\frac{1}{4 \pi R}\left\|J_{1, p, R}\right\|_{2}^{2} \leq & \frac{C_{H_{0}}^{p}}{p !}\left[\int _ { [ 0 , s ] ^ { p } } d \boldsymbol { s } _ { \boldsymbol { p } } \left[\int_{\mathbb{R}^{p}} \mu\left(d \boldsymbol{\xi}_{\boldsymbol{p}}\right) \ell_{R}\left(\tau\left(\boldsymbol{\xi}_{\boldsymbol{p}}\right)\right)\right.\right. \\
& \left.\left.\times\left\{\mathbb{E}\left[e^{-\mathbf{i} \sum_{j=1}^{p}\left(B_{s}-B_{r_{j}}\right) \xi_{j}}\right]-\mathbb{E}\left[e^{-\mathbf{i} \sum_{j=1}^{p}\left(B_{t}-B_{r_{j}}\right) \xi_{j}}\right]\right\}\right]^{\frac{1}{2 H_{0}}}\right]^{2 H_{0}} .
\end{aligned}
$$


We deduce from (5.5) and $\ell_{R}(x)=\frac{\sin ^{2}(R x)}{\pi R x^{2}}$ that

$$
\begin{aligned}
& \frac{1}{4 \pi R}\left\|J_{1, p, R}\right\|_{2}^{2} \\
& \leq \frac{C_{H_{0}}^{p}}{p !} \frac{t-s}{2 \pi T}\left[\int_{[0, s]^{p}} d \boldsymbol{s}_{\boldsymbol{p}}\left(\int_{\mathbb{R}^{p}} \mu\left(d \boldsymbol{\xi}_{\boldsymbol{p}}\right) \mathbb{E}\left[e^{-\mathbf{i} \sum_{j=1}^{p}\left(B_{s}-B_{s_{j}}\right) \xi_{j}}\right]\right)^{\frac{1}{2 H_{0}}}\right]^{2 H_{0}} \\
& =\frac{C_{H_{0}}^{p}}{p !} \frac{t-s}{2 \pi T}\left[K_{2, p}(1, s)\right]^{2 H_{0}} .
\end{aligned}
$$

Then, applying the estimate (3.9) yields

$$
\frac{1}{R}\left\|J_{1, p, R}\right\|_{2}^{2} \leq \frac{2(t-s)}{T}(p !)^{2 H_{0}-1}\left(C_{H_{0}} C_{2}^{2 H_{0}}\right)^{p}\left(\frac{T^{p \mathfrak{h}}}{\Gamma(p \mathfrak{h}+1)}\right)^{2 H_{0}},
$$

for $s<t \leq T \leq R$. Hence, for $s<t \leq T \leq R$,

$$
\begin{aligned}
\frac{1}{\sqrt{R}} \sum_{p=2}^{\infty}\left\|J_{1, p, R}\right\|_{k} & \leq \frac{1}{\sqrt{R}} \sum_{p=2}^{\infty}\left\|J_{1, p, R}\right\|_{2}(k-1)^{p / 2} \\
& \leq \sqrt{\frac{2}{T}}(t-s)^{1 / 2} \sum_{p=2}^{\infty}\left(C_{H_{0}} C_{2}^{2 H_{0}}\right)^{p / 2}(p !)^{\frac{2 H_{0}-1}{2}}\left(\frac{T^{p \mathfrak{h}}}{\Gamma(p \mathfrak{h}+1)}\right)^{H_{0}} \\
& \leq C(t-s)^{1 / 2}
\end{aligned}
$$

where the last inequality follows from the fact that $\frac{2 H_{0}-1}{2}-\mathfrak{h} H_{0}=-\frac{H_{1}}{2}$. From inequality (5.6) together with the one for $p=1$, we can obtain (5.3) in case (H2).

Therefore, in both cases (H1) and (H2), the estimates (5.2) and (5.3) yield (5.1), and hence the proof of tightness is concluded.

\section{Proof of Proposition 1.3}

For $a>0$, we define $\varphi_{a}(t)=\frac{1}{a} \mathbf{1}_{[0, a]}(t), t \in \mathbb{R}$, so $\left\{\varphi_{a}(*) G(\varepsilon, \cdot): \varepsilon, a>0\right\}$ is an approximation to the delta function. Consider the approximating equation

$$
\frac{\partial u^{\varepsilon, a}}{\partial t}(t, x)=\frac{1}{2} \Delta u^{\varepsilon, a}(t, x)+u^{\varepsilon, a}(t, x) \diamond W_{t, x}^{\varepsilon, a},
$$

with initial condition $u^{\varepsilon, a}(t, x)=1$ and

$$
W_{t, x}^{\varepsilon, a}=\int_{0}^{t} \int_{\mathbb{R}} \varphi_{a}(t-s) G(\varepsilon, x-y) W(d s, d y) .
$$

By following exactly the same lines as in the proof of Hu and Nualart (2009, Proposition 5.2), we can show that

$$
u^{\varepsilon, a}(t, x)=\mathbb{E}_{B}\left[\exp \left(W\left(A_{t, x}^{\varepsilon, a, B}\right)-\frac{1}{2}\left\|A_{t, x}^{\varepsilon, a}\right\|_{\mathfrak{H}}^{2}\right)\right]
$$

solves equation (6.1), where $\mathbb{E}_{B}$ denotes the expectation with respect to the randomness $B$ and

$$
\begin{aligned}
A_{t, x}^{\varepsilon, a, B}(r, y) & =\int_{0}^{t} \varphi_{a}(t-s-r) G\left(\varepsilon, B_{s}+x-y\right) d s \mathbf{1}_{[0, t]}(r) \\
& =\frac{1}{a} \int_{0}^{a \wedge(t-r)} G\left(\varepsilon, B_{t-r-s}+x-y\right) d s \mathbf{1}_{[0, t]}(r),
\end{aligned}
$$


with $\left\{B_{s}, s \in \mathbb{R}_{+}\right\}$a standard Brownian motion on $\mathbb{R}$ independent of $W$. We omit the details of the proof of (6.2)

The proof of the Feynman-Kac formula begins with the following identity: For any $t_{1}, \ldots, t_{k}$ and any $x_{1}, \ldots x_{k}$ with $k \geq 2$,

$$
\mathbb{E}\left(\prod_{j=1}^{k} u^{\varepsilon, a}\left(t_{j}, x_{j}\right)\right)=\mathbb{E}\left[\exp \left(\sum_{1 \leq i<j \leq k}\left\langle A_{t_{i}, x_{i}}^{\varepsilon, a, B^{i}}, A_{t_{j}, x_{j}}^{\varepsilon, a, B^{j}}\right\rangle_{\mathfrak{H}}\right)\right]
$$

where $B^{1}, \ldots, B^{k}$ are $k$ i.i.d copies of $B$; see e.g. equation (3.22) in Hu et al. (2015, page 15).

The remaining proof consists of three steps. In Step 1, we will prove that the expectations in (6.3) are uniformly bounded over $\varepsilon>0, a \in\left(0, c_{0}\right)$ for some $c_{0}>0$; the second step is devoted to proving that

$$
\left\langle A_{t_{i}, x_{i}}^{\varepsilon, a, B^{i}}, A_{t_{j}, x_{j}}^{\varepsilon, a, B^{j}}\right\rangle_{\mathfrak{H}} \underset{a . s .}{\stackrel{a \downarrow 0}{\longrightarrow}} \mathcal{I}_{t_{i}, t_{j}, \varepsilon}^{i, j}\left(x_{i}-x_{j}\right) \underset{L^{p}(\Omega)}{\stackrel{\varepsilon \downarrow 0}{\longrightarrow}} \mathcal{I}_{t_{i}, t_{j}}^{i, j}\left(x_{i}-x_{j}\right),
$$

where $\mathcal{I}_{t_{i}, t_{j}, \varepsilon}^{i, j}\left(x_{i}-x_{j}\right)$ and $\mathcal{I}_{t_{i}, t_{j}}^{i, j}\left(x_{i}-x_{j}\right)$ are respective limits whose expressions will be clear later; in Step 3, we will show the $L^{p}(\Omega)$-convergence of $u^{\varepsilon, a}(t, x)$ to $u(t, x)$ as $\varepsilon, a \downarrow 0$. Combining these steps yields

$$
\mathbb{E}\left(\prod_{j=1}^{k} u\left(t_{j}, x_{j}\right)\right)=\mathbb{E}\left[\exp \left(\sum_{1 \leq i<j \leq k} \mathcal{I}_{t_{i}, t_{j}}^{i, j}\left(x_{i}-x_{j}\right)\right)\right]
$$

which is formula (1.8).

Step 1: It suffices to show that for $i<j$ and any $\lambda>0$,

$$
\sup \left\{\mathbb{E}\left[\exp \left(\lambda\left|\left\langle A_{t_{i}, x_{i}}^{\varepsilon, a, B^{i}}, A_{t_{j}, x_{j}}^{\varepsilon, a, B_{j}^{j}}\right\rangle_{\mathfrak{H}}\right|\right)\right]: a \in\left(0, t_{i} \wedge t_{j}\right], \varepsilon>0\right\}<+\infty .
$$

Similar to (3.23) and (3.24) in Hu et al. (2015, page 15), we have

$$
\begin{aligned}
\left\langle A_{t_{i}, x_{i}}^{\varepsilon, a, B^{i}}, A_{t_{j}, x_{j}}^{\varepsilon, a, B^{j}}\right\rangle_{\mathfrak{H}}= & \int_{\mathbb{R}} \mu(d \xi) e^{-\varepsilon \xi^{2}} e^{-\mathbf{i} \xi\left(x_{i}-x_{j}\right)} \int_{0}^{t_{i}} \int_{0}^{t_{j}} d r d \widetilde{r} \gamma_{0}\left(t_{i}-r-t_{j}+\widetilde{r}\right) \\
& \times \frac{1}{a^{2}} \int_{0}^{a \wedge r} \int_{0}^{a \wedge \widetilde{r}} d s d \tilde{s} e^{-\mathbf{i} \xi\left(B_{r-s}^{i}-B_{\widetilde{r}-\tilde{s}}^{j}\right)}
\end{aligned}
$$


Thus, with $m=2 n \geq 2$, we can write

$$
\begin{aligned}
& \mathbb{E}\left[\left\langle A_{t_{i}, x_{i}}^{\varepsilon, a, B^{i}}, A_{t_{j}, x_{j}}^{\varepsilon, a, B^{j}}\right\rangle_{\mathfrak{H}}^{m}\right] \\
= & \int_{\mathbb{R}^{m}} \mu\left(d \boldsymbol{\xi}_{\boldsymbol{m}}\right)\left(\prod_{k=1}^{m} e^{-\varepsilon \xi_{k}^{2}} e^{-\mathbf{i} \xi_{k}\left(x_{i}-x_{j}\right)}\right) \int_{\left[0, t_{i}\right]^{m} \times\left[0, t_{j}\right]^{m}} d \boldsymbol{r}_{\boldsymbol{m}} d \widetilde{\boldsymbol{r}}_{\boldsymbol{m}} \\
& \times\left(\prod_{k=1}^{m} \gamma_{0}\left(t_{i}-r_{k}-t_{j}+\widetilde{r}_{k}\right)\right) \frac{1}{a^{2 m}} \int_{[0, a]^{2 m}} d \boldsymbol{s}_{\boldsymbol{m}} d \widetilde{\boldsymbol{s}}_{\boldsymbol{m}} \mathbf{1}_{\left\{s_{k} \leq r_{k}, \widetilde{s}_{k} \leq \widetilde{r}_{k} ; \forall k\right\}} \\
& \times \mathbb{E}\left[\exp \left(-\mathbf{i} \sum_{k=1}^{m} \xi_{k}\left(B_{r_{k}-s_{k}}^{i}-B_{\widetilde{r}_{k}-\widetilde{s}_{k}}^{j}\right)\right)\right] \\
\leq & \frac{1}{a^{2 m}} \int_{[0, a]^{2 m}} d \boldsymbol{s}_{\boldsymbol{m}} d \widetilde{\boldsymbol{s}}_{\boldsymbol{m}} \int_{\mathbb{R}^{m}} \mu\left(d \boldsymbol{\xi}_{\boldsymbol{m}}\right) \int_{\left[0, t_{i}\right]^{m} \times\left[0, t_{j}\right]^{m}} d \boldsymbol{r}_{\boldsymbol{m}} d \widetilde{\boldsymbol{r}}_{\boldsymbol{m}} \mathbf{1}_{\left\{s_{k} \leq r_{k}, \widetilde{s}_{k} \leq \widetilde{r}_{k} ; \forall k\right\}} \\
& \times\left(\prod_{k=1}^{m} \gamma_{0}\left(t_{i}-r_{k}-t_{j}+\widetilde{r}_{k}\right)\right) \mathbb{E}\left[\exp \left(-\mathbf{i} \sum_{k=1}^{m} \xi_{k}\left(B_{r_{k}-s_{k}}^{i}-B_{\widetilde{r}_{k}-\widetilde{s}_{k}}^{j}\right)\right)\right]
\end{aligned}
$$

and for $s_{k} \leq r_{k}, \widetilde{s}_{k} \leq \widetilde{r}_{k}$

$$
\begin{aligned}
& \mathbb{E}\left[\exp \left(-\mathbf{i} \sum_{k=1}^{m} \xi_{k}\left(B_{r_{k}-s_{k}}^{i}-B_{\widetilde{r}_{k}-\widetilde{s}_{k}}^{j}\right)\right)\right] \\
= & \exp \left(-\frac{1}{2} \operatorname{Var} \sum_{k=1}^{m} \xi_{k}\left(B_{r_{k}-s_{k}}^{i}-B_{\widetilde{r_{k}}-\widetilde{s}_{k}}^{j}\right)\right) \leq \exp \left(-\frac{1}{2} \operatorname{Var} \sum_{k=1}^{m} \xi_{k} B_{r_{k}-s_{k}}^{i}\right) .
\end{aligned}
$$

Now let us consider case (H1) where $\gamma_{0}$ is locally integrable and the spectral density $\varphi$ satisfies the modified Dalang's condition (D). By changing $\left(r_{k}-s_{k}, \widetilde{r}_{k}-\widetilde{s}_{k}\right)$ to $\left(r_{k}, \widetilde{r}_{k}\right)$ for each $k$, we get the following estimate $\left(a \leq t_{i} \wedge t_{j}\right)$

$$
\begin{aligned}
& \mathbb{E}\left[\left\langle A_{t_{i}, x_{i}}^{\varepsilon, a, B^{i}}, A_{t_{j}, x_{j}}^{\varepsilon, a, B^{j}}\right\rangle_{\mathfrak{H}}^{m}\right] \leq \frac{1}{a^{2 m}} \int_{[0, a]^{2 m}} d \boldsymbol{s}_{\boldsymbol{m}} d \widetilde{\boldsymbol{s}}_{\boldsymbol{m}} \int_{\mathbb{R}^{m}} \mu\left(d \boldsymbol{\xi}_{\boldsymbol{m}}\right) \int_{\left[0, t_{i}\right]^{m} \times\left[0, t_{j}\right]^{m}} d \boldsymbol{r}_{\boldsymbol{m}} d \widetilde{\boldsymbol{r}}_{\boldsymbol{m}} \\
& \quad \times\left(\prod_{k=1}^{m} \gamma_{0}\left(t_{i}-r_{k}-s_{k}-t_{j}+\widetilde{r}_{k}+\widetilde{s}_{k}\right)\right) \exp \left(-\frac{1}{2} \operatorname{Var} \sum_{k=1}^{m} \xi_{k} B_{r_{k}}^{i}\right) \\
& \leq \Gamma_{t_{i}+t_{j}}^{m} \int_{\mathbb{R}^{m}} \mu\left(d \boldsymbol{\xi}_{\boldsymbol{m}}\right) \int_{\left[0, t_{i}\right]^{m}} d \boldsymbol{r}_{\boldsymbol{m}} \exp \left(-\frac{1}{2} \operatorname{Var} \sum_{k=1}^{m} \xi_{k} B_{r_{k}}\right)=\Gamma_{t_{i}+t_{j}}^{m} K_{1, m}\left(1, t_{i}\right),
\end{aligned}
$$

with $\Gamma_{t}:=\int_{-t}^{t} \gamma_{0}(s) d s$ and $K_{1, m}\left(1, t_{i}\right)$ defined as in (3.1). The estimate (3.7) yields

$$
\mathbb{E}\left[\left\langle A_{t_{i}, x_{i}}^{\varepsilon, a, B^{i}}, A_{t_{j}, x_{j}}^{\varepsilon, a, B^{j}}\right\rangle_{\mathfrak{H}}^{m}\right] \leq \frac{1}{2 \kappa_{0}} m !\left(8 \kappa_{0} C_{N} \Gamma_{t_{i}+t_{j}}\right)^{m} e^{\frac{t_{i} D_{N}}{2 C_{N}}}
$$

In particular, for any $n \in \mathbb{N}$, we have

$$
\mathbb{E}\left[\left|\left\langle A_{t_{i}, x_{i}}^{\varepsilon, a, B^{i}}, A_{t_{j}, x_{j}}^{\varepsilon, a, B^{j}}\right\rangle_{\mathfrak{H}}\right|^{n}\right] \leq\left(2 \kappa_{0}\right)^{-1 / 2} \sqrt{(2 n) ! e^{\frac{t_{i} D_{N}}{2 C_{N}}}}\left(8 \kappa_{0} C_{N} \Gamma_{t_{i}+t_{j}}\right)^{n} .
$$


Note that for any given $\lambda \in(0, \infty)$ we can choose $N>0$ such that $0<16 \kappa_{0} C_{N} \Gamma_{t_{i}+t_{j}} \lambda<1$. Thus, we deduce that

$$
\begin{aligned}
& \mathbb{E}\left[\exp \left(\lambda\left|\left\langle A_{t_{i}, x_{i}}^{\varepsilon, a, B^{i}}, A_{t_{j}, x_{j}}^{\varepsilon, a, B^{j}}\right\rangle_{\mathfrak{H}}\right|\right)\right] \\
& \leq 1+\sum_{n=1}^{\infty} \frac{\lambda^{n}}{n !} \mathbb{E}\left[\left|\left\langle A_{t_{i}, x_{i}}^{\varepsilon, a, B^{i}}, A_{t_{j}, x_{j}}^{\varepsilon, a, B^{j}}\right\rangle_{\mathfrak{H}}\right|^{n}\right] \\
& \leq 1+\left(2 \kappa_{0}\right)^{-1 / 2} \sqrt{e^{\frac{t_{i} D_{N}}{2 C_{N}}}} \sum_{n=1}^{\infty} \frac{\sqrt{(2 n) !}}{n !}\left(8 \lambda \kappa_{0} C_{N}^{\prime} \Gamma_{t_{i}+t_{j}}\right)^{n} \\
& \leq 1+\left(2 \kappa_{0}\right)^{-1 / 2} \sqrt{e^{\frac{t_{i} D_{N}}{2 C_{N}}}} \sum_{n=1}^{\infty}\left(16 \lambda \kappa_{0} C_{N}^{\prime} \Gamma_{t_{i}+t_{j}}\right)^{n},
\end{aligned}
$$

where we used $(2 n) ! \leq 4^{n}(n !)^{2}$. Hence, we have proved (6.4) in case $(\mathbf{H 1})$.

Now let us consider case (H2) where $\gamma_{0}(t)=|t|^{2 H_{0}-2}$ for some $H_{0} \in(1 / 2,1)$ and $\varphi(x)=|x|^{1-2 H_{1}}$ for some $H_{1} \in(0,1 / 2)$ such that $H_{0}+H_{1}>3 / 4$. We begin with (6.5) and make the change of variables $t_{i}-r_{k} \rightarrow r_{k}$ and $t_{j}-\widetilde{r}_{k} \rightarrow \widetilde{r}_{k}$, to write, with $m=2 n \geq 2$,

$$
\begin{aligned}
& \mathbb{E}\left[\left\langle A_{t_{i}, x_{i}}^{\varepsilon, a, B^{i}}, A_{t_{j}, x_{j}}^{\varepsilon, a, B^{j}}\right\rangle_{\mathfrak{H}}^{m}\right] \\
& \leq \frac{1}{a^{2 m}} \int_{[0, a]^{2 m}} d \boldsymbol{s}_{\boldsymbol{m}} d \widetilde{\boldsymbol{s}}_{\boldsymbol{m}} \int_{\mathbb{R}^{m}} \mu\left(d \boldsymbol{\xi}_{\boldsymbol{m}}\right) \int_{\left[0, t_{i}\right]^{m} \times\left[0, t_{j}\right]^{m}} d \boldsymbol{r}_{\boldsymbol{m}} d \widetilde{\boldsymbol{r}}_{\boldsymbol{m}} \\
& \times \mathbf{1}_{\left\{s_{k} \leq t_{i}-r_{k}, \widetilde{s}_{k} \leq t_{j}-\widetilde{r}_{k} ; \forall k\right\}}\left(\prod_{k=1}^{m}\left|r_{k}-\widetilde{r}_{k}\right|^{2 H_{0}-2}\right) \\
& \left.\times \exp \left(-\operatorname{Var} \sum_{k=1}^{m} \xi_{k} B_{t_{i}-r_{k}-s_{k}}\right) \exp \left(-\operatorname{Var} \sum_{k=1}^{m} B_{t_{j}-\widetilde{r}_{k}-\widetilde{s}_{k}}\right)\right) .
\end{aligned}
$$

Then, the embedding property (3.23), together with Cauchy-Schwarz inequality and the change of variables $v_{k}=t_{i}-r_{k}-s_{k}, \widetilde{v}_{k}=t_{j}-\widetilde{r}_{k}-\widetilde{s}_{k}$, leads to

$$
\begin{aligned}
\mathbb{E} & {\left[\left\langle A_{t_{i}, x_{i}}^{\varepsilon, a, B^{i}}, A_{t_{j}, x_{j}}^{\varepsilon, a, B^{j}}\right\rangle_{\mathfrak{H}}^{m}\right] } \\
\leq & C_{H_{0}}^{m}\left(\int_{\left[0, t_{i}\right]^{m}} d \boldsymbol{v}_{\boldsymbol{m}}\left(\int_{\mathbb{R}^{m}} \mu\left(d \boldsymbol{\xi}_{\boldsymbol{m}}\right) \exp \left(-\operatorname{Var} \sum_{k=1}^{m} \xi_{k} B_{v_{k}}^{i}\right)\right)^{\frac{1}{2 H_{0}}}\right)^{H_{0}} \\
& \times\left(\int_{\left[0, t_{j}\right]^{m}} d \boldsymbol{v}_{\boldsymbol{m}}\left(\int_{\mathbb{R}^{m}} \mu\left(d \boldsymbol{\xi}_{\boldsymbol{m}}\right) \exp \left(-\operatorname{Var} \sum_{k=1}^{m} \xi_{k} B_{v_{k}}^{i}\right)\right)^{\frac{1}{2 H_{0}}}\right)^{H_{0}} \\
= & C_{H_{0}}^{m}\left[K_{2, m}\left(1, t_{i}\right) K_{2, m}\left(1, t_{j}\right)\right]^{H_{0}}
\end{aligned}
$$

Finally, using the estimate (3.9), we can write

$$
\mathbb{E}\left[\left\langle A_{t_{i}, x_{i}}^{\varepsilon, a, B^{i}}, A_{t_{j}, x_{j}}^{\varepsilon, a, B^{j}}\right\rangle_{\mathfrak{H}}^{m}\right] \leq C_{H_{0}}^{m} C_{2}^{2 H_{0} m}\left(t_{i} t_{j}\right)^{m \mathfrak{h}} \frac{(m !)^{2 H_{0}}}{(\Gamma(m \mathfrak{h}+1))^{2 H_{0}}}
$$


Thus, for any $\lambda>0$,

$$
\begin{aligned}
\mathbb{E}\left[e^{\lambda\left|\left\langle A_{t_{i}, x_{i}}^{\varepsilon, a, B^{i}}, A_{t_{j}, x_{j}}^{\varepsilon, a, B^{j}}\right\rangle_{\mathfrak{H}}\right|}\right] & \leq 2+2 \sum_{n=1}^{\infty} \frac{\lambda^{2 n}}{(2 n) !} \mathbb{E}\left[\left|\left\langle A_{t_{i}, x_{i}}^{\varepsilon, a, B^{i}}, A_{t_{j}, x_{j}}^{\varepsilon, a, B^{j}}\right\rangle_{\mathfrak{H}}\right|^{2 n}\right] \\
& \leq 2+2 \sum_{n=1}^{\infty}\left[\lambda C_{H_{0}} C_{2}^{2 H_{0}}\left(t_{i} t_{j}\right)^{\mathfrak{h}}\right]^{2 n} \frac{((2 n) !)^{2 H_{0}-1}}{(\Gamma(2 n \mathfrak{h}+1))^{2 H_{0}}}<\infty
\end{aligned}
$$

because $2 H_{0}-1-2 H_{0} \mathfrak{h}=-H_{1}<0$. This implies (6.4) in case (H2).

Step 2. For fixed $\varepsilon>0$ and $i<j$, as $a \downarrow 0$,

$$
\frac{1}{a^{2}} \int_{0}^{a \wedge r} \int_{0}^{a \wedge \widetilde{r}} d s_{1} d s_{2} e^{-\mathbf{i} \xi\left(B_{r-s_{1}}^{i}-B_{\widetilde{r}-s_{2}}^{j}\right)}
$$

is uniformly bounded and converges to $e^{-\mathbf{i} \xi\left(B_{r}^{i}-B_{\widetilde{r}}^{j}\right)}$, due to the path continuity of Brownian motion. As $\mu_{\varepsilon}(d \xi)=e^{-\varepsilon \xi^{2}} \mu(d \xi)$ is a finite measure and $\gamma_{0}$ is locally integrable, we have

$$
\begin{gathered}
\left\langle A_{t_{i}, x_{i}}^{\varepsilon, a, B^{i}}, A_{t_{j}, x_{j}}^{\varepsilon, a, B^{j}}\right\rangle_{\mathfrak{H}} \underset{a \downarrow 0}{\stackrel{a . s .}{\longrightarrow}} \int_{\mathbb{R}} \mu(d \xi) e^{-\varepsilon \xi^{2}-\mathbf{i} \xi\left(x_{i}-x_{j}\right)} \int_{0}^{t_{i}} \int_{0}^{t_{j}} d r d \widetilde{r} \gamma_{0}\left(t_{i}-r-t_{j}+\widetilde{r}\right) \\
\times e^{-\mathbf{i} \xi\left(B_{r}^{i}-B_{\widetilde{r}}^{j}\right)}=: \mathcal{I}_{t_{i}, t_{j}, \varepsilon}^{i, j}\left(x_{i}-x_{j}\right) .
\end{gathered}
$$

The above almost sure limit $\mathcal{I}_{t_{i}, t_{j}, \varepsilon}^{i, j}\left(x_{i}-x_{j}\right)$ is real for any $x_{i}, x_{j} \in \mathbb{R}$ and any $\varepsilon>0$, since $\mu$ is symmetric (i.e. the spectral density is an even function on $\mathbb{R}$ ). In the sequel, we just write $\mathcal{I}_{\varepsilon}^{i, j}\left(x_{i}-x_{j}\right), \mathcal{I}^{i, j}\left(x_{i}-x_{j}\right)$ to mean $\mathcal{I}_{t_{i}, t_{j}, \varepsilon}^{i, j}\left(x_{i}-x_{j}\right)$ and $\mathcal{I}_{t_{i}, t_{j}}^{i, j}\left(x_{i}-x_{j}\right)$ respectively.

We will prove $\mathcal{I}_{\varepsilon}^{i, j}\left(x_{i}-x_{j}\right)$ converges, as $\varepsilon \downarrow 0$, in $L^{2}(\Omega)$ (hence in probability) to some limit, denoted by $\mathcal{I}^{i, j}\left(x_{i}-x_{j}\right)$. Note that, by Fatou's lemma and the estimates in Step 1, we can establish that for any $\lambda \in \mathbb{R}$,

$$
\mathbb{E}\left[\exp \left(\lambda \mathcal{I}_{\varepsilon}^{i, j}\left(x_{i}-x_{j}\right)\right)\right] \leq C_{\lambda}
$$

for all $\varepsilon>0$, where $C_{\lambda}$ is a constant that does not depend on $\varepsilon$. Now we rewrite

$$
\mathcal{I}_{\varepsilon}^{i, j}\left(x_{i}-x_{j}\right)=\int_{\mathbb{R}} d \xi \varphi(\xi) e^{-\varepsilon \xi^{2}} \int_{0}^{t_{i}} \int_{0}^{t_{j}} d r d \widetilde{r} \gamma_{0}(r-\widetilde{r}) e^{-\mathbf{i} \xi\left(B_{t_{i}-r}^{i}-B_{t_{j}-\widetilde{r}}^{j}+x_{i}-x_{j}\right)}
$$

and we compute for $\varepsilon_{1}, \varepsilon_{2}>0$,

$$
\begin{aligned}
\mathbb{E}\left[\mathcal{I}_{\varepsilon_{1}}^{i, j}\left(x_{i}-x_{j}\right) \mathcal{I}_{\varepsilon_{2}}^{i, j}\left(x_{i}-x_{j}\right)\right]= & \int_{\mathbb{R}^{2}} d \boldsymbol{\xi}_{\mathbf{2}} \varphi\left(\xi_{1}\right) \varphi\left(\xi_{2}\right) e^{-\sum_{k=1}^{2} \varepsilon_{k} \xi_{k}^{2}} e^{-\mathbf{i}\left(x_{i}-x_{j}\right)\left(\xi_{1}+\xi_{2}\right)} \\
& \times \int_{\left[0, t_{i}\right]^{2} \times\left[0, t_{j}\right]^{2}} d \boldsymbol{r}_{\mathbf{2}} d \widetilde{\boldsymbol{r}}_{\mathbf{2}} \gamma_{0}\left(r_{1}-\widetilde{r}_{1}\right) \gamma_{0}\left(r_{2}-\widetilde{r}_{2}\right) \\
& \times \mathbb{E}\left[e^{-\mathbf{i} \sum_{k=1}^{2} \xi_{k}\left(B_{t_{i}-r_{k}}^{i}-B_{t_{j}-\widetilde{r}_{k}}^{j}\right)}\right]
\end{aligned}
$$

Note that $\mathbb{E}\left[e^{-\mathbf{i} \sum_{k=1}^{2} \xi_{k}\left(B_{t_{i}-r_{k}}^{i}-B_{t_{j}-\widetilde{r}_{k}}^{j}\right)}\right]=\exp \left[-\frac{1}{2} \operatorname{Var} \sum_{k=1}^{2} \xi_{k}\left(B_{t_{i}-r_{k}}^{i}-B_{t_{j}-\widetilde{r}_{k}}^{j}\right)\right]$ is positive, and note also from previous calculations in both cases (H1) and (H2) 
that

$$
\begin{gathered}
\int_{\mathbb{R}^{2}} d \xi_{1} d \xi_{2} \varphi\left(\xi_{1}\right) \varphi\left(\xi_{2}\right) \int_{\left[0, t_{i}\right]^{2} \times\left[0, t_{j}\right]^{2}} d \boldsymbol{r}_{\mathbf{2}} d \widetilde{\boldsymbol{r}}_{\mathbf{2}} \gamma_{0}\left(r_{1}-\widetilde{r}_{1}\right) \gamma_{0}\left(r_{2}-\widetilde{r}_{2}\right) \\
\quad \times \exp \left(-\frac{1}{2} \operatorname{Var} \sum_{k=1}^{2} \xi_{k}\left(B_{t_{i}-r_{k}}^{i}-B_{t_{j}-\widetilde{r}_{k}}^{j}\right)\right)<\infty .
\end{gathered}
$$

By the dominated convergence theorem, the limit

$$
\lim _{\varepsilon_{1}, \varepsilon_{2} \downarrow 0} \mathbb{E}\left[\mathcal{I}_{\varepsilon_{1}}^{i, j}\left(x_{i}-x_{j}\right) \mathcal{I}_{\varepsilon_{2}}^{i, j}\left(x_{i}-x_{j}\right)\right]
$$

exists. Therefore, as $\varepsilon \downarrow 0, \mathcal{I}_{\varepsilon}^{i, j}\left(x_{i}-x_{j}\right)$ converges in $L^{2}(\Omega)$ to some limit $\mathcal{I}^{i, j}\left(x_{i}-\right.$ $x_{j}$ ), which is formally given by

$$
\mathcal{I}^{i, j}\left(x_{i}-x_{j}\right)=\int_{\mathbb{R}} d \xi \varphi(\xi) \int_{0}^{t_{i}} \int_{0}^{t_{j}} d r d \widetilde{r} \gamma_{0}(r-\widetilde{r}) e^{-\mathbf{i} \xi\left(B_{t_{i}-r}^{i}-B_{t_{j}-\widetilde{r}}^{j}+x_{i}-x_{j}\right)} .
$$

In addition, it is easy to show that this convergence also takes place in $L^{p}(\Omega)$ for any $p \geq 1$.

Thus, together with (6.4), we deduce by first passing $a$ to zero, then $\varepsilon$ to zero that

$$
\mathbb{E}\left[\exp \left(\sum_{1 \leq i<j \leq k}\left\langle A_{t_{i}, x_{i}}^{\varepsilon, a, B^{i}}, A_{t_{j}, x_{j}}^{\varepsilon, a, B^{j}}\right\rangle_{\mathfrak{H}}\right)\right] \rightarrow \mathbb{E}\left[\exp \left(\sum_{1 \leq i<j \leq k} \mathcal{I}^{i, j}\left(x_{i}-x_{j}\right)\right)\right] .
$$

Step 3. The last step is to establish the $L^{p}(\Omega)$-convergence of $u^{\varepsilon, a}(t, x)$ to $u(t, x)$, as $\varepsilon, a \downarrow 0$. It is enough to show the $L^{2}(\Omega)$-convergence in view of the moment bounds from Step 1. First for $\varepsilon_{1}, \varepsilon_{2}, a_{1}, a_{2}>0$, we can write by similar arguments as before,

$$
\mathbb{E}\left[u^{\varepsilon_{1}, a_{1}}(t, x) u^{\varepsilon_{2}, a_{2}}(t, x)\right]=\mathbb{E}\left[\exp \left(\left\langle A_{t, x}^{\varepsilon_{1}, a_{1}, B^{1}}, A_{t, x}^{\varepsilon_{2}, a_{2}, B^{2}}\right\rangle_{\mathfrak{H}}\right)\right]
$$

and

$$
\begin{aligned}
&\left\langle A_{t, x}^{\varepsilon_{1}, a_{1}, B^{1}}, A_{t, x}^{\varepsilon_{2}, a_{2}, B^{2}}\right\rangle_{\mathfrak{H}}=\int_{\mathbb{R}} \mu(d \xi) e^{-\frac{\varepsilon_{1}+\varepsilon_{2}}{2} \xi^{2}} \int_{[0, t]^{2}} d r d \widetilde{r} \gamma_{0}(r-\widetilde{r}) \frac{1}{a_{1} a_{2}} \\
& \times \int_{0}^{a_{1} \wedge r} \int_{0}^{a_{2} \wedge \widetilde{r}} d s_{1} d s_{2} e^{-\mathbf{i} \xi\left(B_{r-s_{1}}^{1}-B_{\widetilde{r}-s_{2}}^{2}\right)} \\
& \stackrel{a_{1}, a_{2} \downarrow 0}{a . s .} \int_{\mathbb{R}} \mu(d \xi) e^{-\frac{\varepsilon_{1}+\varepsilon_{2}}{2} \xi^{2}} \int_{[0, t]^{2}} d r d \widetilde{r} \gamma_{0}(r-\widetilde{r}) e^{-\mathbf{i} \xi\left(B_{r}^{1}-B_{\widetilde{r}}^{2}\right)} \frac{\varepsilon_{1}, \varepsilon_{2} \downarrow 0}{i n L^{2}(\Omega)} \mathcal{I}_{t, t}^{1,2}(0) .
\end{aligned}
$$

By (6.4) again, we have

$$
\mathbb{E}\left[u^{\varepsilon_{1}, a_{1}}(t, x) u^{\varepsilon_{2}, a_{2}}(t, x)\right] \underset{\text { then } \varepsilon_{1}, \varepsilon_{2} \downarrow 0}{\stackrel{a_{1}, a_{2} \downarrow 0}{\longrightarrow}} \mathbb{E}\left[e^{\mathcal{I}_{t, t}^{1,2}(0)}\right],
$$

which implies that the limit

$$
v(t, x):=\lim _{\varepsilon \downarrow 0} \lim _{a \downarrow 0} u^{\varepsilon, a}(t, x)
$$

exists in $L^{p}(\Omega)$ for any $p \geq 1$.

Now consider a test random variable $F=\exp \left(W(g)-\frac{1}{2}\|g\|_{\mathfrak{H}}^{2}\right)$ for $g \in C_{c}^{\infty}\left(\mathbb{R}_{+} \times\right.$ $\mathbb{R})$ and recall that random variables of this from are dense in $\mathbb{D}^{1,2}$. For such a $F$, 
we have

$$
\begin{aligned}
\mathbb{E}\left[F u^{\varepsilon, a}(t, x)\right] & =\mathbb{E}\left[\exp \left(W\left(A_{t, x}^{\varepsilon, a, B}+g\right)-\frac{1}{2}\left\|A_{t, x}^{\varepsilon, a, B}\right\|_{\mathfrak{H}}^{2}-\frac{1}{2}\|g\|_{\mathfrak{H}}^{2}\right)\right] \\
& =\mathbb{E}\left[\exp \left(\left\langle A_{t, x}^{\varepsilon, a, B}, g\right\rangle_{\mathfrak{H}}\right)\right] \\
& =\mathbb{E}\left[\exp \left(\int_{0}^{t}\left\langle\varphi_{a}(s-\bullet) G\left(\varepsilon, B_{t-s}+x-*\right), g(\bullet, *)\right\rangle_{\mathfrak{H}} d s\right)\right],
\end{aligned}
$$

then putting $S_{t, x}=\mathbb{E}\left[F u^{\varepsilon, a}(t, x)\right]$, we deduce from the classical Feynman-Kac formula that $S_{t, x}$ solves the partial differential equation

$$
\partial_{t} S_{t, x}=\frac{1}{2} \Delta S_{t, x}+S_{t, x}\left\langle\varphi_{a}(t-\bullet) G_{\varepsilon}(x-*), g(\bullet, *)\right\rangle_{\mathfrak{H}}
$$

with initial condition $S_{0, x}=\mathbb{E}[F]=1$; see for instance Hu and Nualart (2009, page $315)$. It follows that

$$
\begin{aligned}
& \mathbb{E}\left[F u^{\varepsilon, a}(t, x)\right] \\
& =1+\int_{0}^{t} \int_{\mathbb{R}} \mathbb{E}\left[F u^{\varepsilon, a}(s, y)\right] G_{t-s}(x-y)\left\langle\varphi_{a}(s-\bullet) G_{\varepsilon}(y-*), g(\bullet, *)\right\rangle_{\mathfrak{H}} d s d y \\
& \underset{\text { then } \varepsilon \downarrow 0}{\longrightarrow a \downarrow 0} 1+\mathbb{E}\left\langle D F, v G_{t-\bullet}(x-*)\right\rangle_{\mathfrak{H}},
\end{aligned}
$$

where the convergence in (6.6) is verified at the end of this proof. Assuming (6.6), we have

$$
\mathbb{E}[F v(t, x)]=1+\mathbb{E}\left\langle D F, v G_{t-\bullet}(x-*)\right\rangle_{\mathfrak{H}},
$$

which is equivalent to say that $v(t, x)$ solves the same equation for $u(t, x)$ so that $v(t, x)=u(t, x)$ by the uniqueness of the mild solution; see also Hu and Nualart (2009) for similar arguments for the case where the Gaussian noise is fractional in time and white in space.

Now, let us now justify the convergence in (6.6). First, by $L^{2}$-convergence of $u^{\varepsilon, a}(s, y)$, we have

$$
\mathbb{E}\left[F u^{\varepsilon, a}(s, y)\right] \underset{\text { then } \varepsilon \downarrow 0}{\stackrel{a \downarrow 0}{\longrightarrow}} \mathbb{E}[F v(s, y)] .
$$

And recall that $g \in C_{c}^{\infty}\left(\mathbb{R}_{+} \times \mathbb{R}\right)\left(\operatorname{suppose} g(r, y)=0\right.$ for $\left.(r, y) \in[0, K]^{c} \times[-K, K]^{c}\right)$, so that

$$
|\mathcal{F} g(r, \xi)| \leq C \wedge \frac{C}{\xi^{2}}, \forall(r, \xi) \in \mathbb{R}_{+} \times \mathbb{R}
$$

Combining the above bound and Dalang's condition, we have

$$
\int_{\mathbb{R}} d \xi \varphi(\xi)|\mathcal{F} g|(\widetilde{r}, \xi) \leq C \int_{\{|\xi| \leq 1\}} \varphi(\xi) d \xi+C \int_{\{|\xi|>1\}} \frac{\varphi(\xi)}{\xi^{2}} d \xi<+\infty .
$$

Noting also that $\gamma_{0}$ is locally integrable, we observe that

$$
\left\langle\varphi_{a}(s-\bullet) G_{\varepsilon}(y-*), g\right\rangle_{\mathfrak{H}}=\int_{\mathbb{R}_{+}^{2} \times \mathbb{R}} d r d \widetilde{r} d \xi \gamma_{0}(r-\widetilde{r}) \varphi_{a}(s-r) \varphi(\xi) e^{-\mathbf{i} y \xi-\frac{\varepsilon}{2} \xi^{2}} \mathcal{F} g(\widetilde{r},-\xi)
$$


is uniformly bounded over $(s, y, \varepsilon, a) \in[0, t] \times \mathbb{R} \times(0, \infty)^{2}$. Hence,

$$
\begin{aligned}
\left\langle\varphi_{a}(s-\bullet) G_{\varepsilon}(y-*), g\right\rangle_{\mathfrak{H}} & =\int_{0}^{K} d \widetilde{r}\left(\varphi_{a} * \gamma_{0}\right)(s-\widetilde{r}) \int_{\mathbb{R}} d \xi \varphi(\xi) e^{-\mathbf{i} y \xi-\frac{\varepsilon}{2} \xi^{2}} \mathcal{F} g(\widetilde{r},-\xi) \\
& \stackrel{a \rightarrow 0}{\longrightarrow} \int_{0}^{K} d \widetilde{r} \gamma_{0}(s-\widetilde{r}) \int_{\mathbb{R}} d \xi \varphi(\xi) e^{-\mathbf{i} y \xi-\frac{\varepsilon}{2} \xi^{2}} \mathcal{F} g(\widetilde{r},-\xi) \\
& \stackrel{\varepsilon \rightarrow 0}{\longrightarrow} \int_{0}^{K} d \widetilde{r} \gamma_{0}(s-\widetilde{r}) \int_{\mathbb{R}} d \xi \varphi(\xi) e^{-\mathbf{i} y \xi} \mathcal{F} g(\widetilde{r},-\xi)
\end{aligned}
$$

where we used (6.7) and the fact that $\varphi_{a} * \gamma_{0}$ converges in $L_{\text {loc }}^{1}(\mathbb{R})$ to $\gamma_{0}$ to obtain (6.8) and we applied the dominated convergence theorem in (6.9).

Another application of dominated convergence together with Fubini's theorem leads to

$$
\begin{aligned}
& \mathbb{E}[F v(t, x)] \\
= & 1+\int_{0}^{t} \int_{\mathbb{R}} \mathbb{E}[F v(s, y)] G_{t-s}(x-y) \int_{\mathbb{R}} d \widetilde{r} \gamma_{0}(s-\widetilde{r}) \int_{\mathbb{R}} d \xi \varphi(\xi) e^{-\mathbf{i} y \xi} \mathcal{F} g(\widetilde{r},-\xi) d s d y \\
= & 1+\mathbb{E} F \int_{0}^{t} d s \int_{\mathbb{R}_{+}} d \widetilde{r} \gamma_{0}(s-\widetilde{r}) \int_{\mathbb{R}} d \xi \varphi(\xi) \mathcal{F}\left(v G_{t-\bullet}(x-*)\right)(s, \xi) \mathcal{F} g(\widetilde{r},-\xi) \\
= & 1+\mathbb{E} F\left\langle g, v G_{t-\bullet}(x-*)\right\rangle_{\mathfrak{H}}=1+\mathbb{E}\left\langle D F, v G_{t-\bullet}(x-*)\right\rangle_{\mathfrak{H}},(\text { as } D F=F g),
\end{aligned}
$$

which confirms the convergence in (6.6).

Therefore, we can conclude our proof by combining the above steps.

\section{References}

Balan, R. M., Jolis, M., and Quer-Sardanyons, L. SPDEs with affine multiplicative fractional noise in space with index $1 / 4<H<1 / 2$. Electron. J. Probab., 20, no. 54, 36 (2015). MR3354614.

Campese, S., Nourdin, I., and Nualart, D. Continuous Breuer-Major theorem: tightness and nonstationarity. Ann. Probab., 48 (1), 147-177 (2020). MR4079433.

Chen, L., Khoshnevisan, D., Nualart, D., and Pu, F. Poincaré inequality and central limit theorems for parabolic stochastic partial differential equations. ArXiv Mathematics e-prints (2019). arXiv: 1912.01482.

Chen, L., Khoshnevisan, D., Nualart, D., and Pu, F. Spatial ergodicity and central limit theorem for parabolic Anderson model with delta initial condition. ArXiv Mathematics e-prints (2020). arXiv: 2005.10417.

Dalang, R. C. Extending the martingale measure stochastic integral with applications to spatially homogeneous s.p.d.e.'s. Electron. J. Probab., 4, no. 6, 29 (1999). MR1684157.

Delgado-Vences, F., Nualart, D., and Zheng, G. A central limit theorem for the stochastic wave equation with fractional noise. Ann. Inst. Henri Poincaré Probab. Stat., 56 (4), 3020-3042 (2020). MR4164864.

$\mathrm{Gu}, \mathrm{Y}$. and Li, J. Fluctuations of a nonlinear stochastic heat equation in dimensions three and higher. SIAM J. Math. Anal., 52 (6), 5422-5440 (2020). MR4169750.

Hu, Y., Huang, J., Lê, K., Nualart, D., and Tindel, S. Stochastic heat equation with rough dependence in space. Ann. Probab., 45 (6B), 4561-4616 (2017). MR3737918. 
Hu, Y., Huang, J., Lê, K., Nualart, D., and Tindel, S. Parabolic Anderson model with rough dependence in space. In Computation and combinatorics in dynamics, stochastics and control, volume 13 of Abel Symp., pp. 477-498. Springer, Cham (2018). MR3967394.

Hu, Y., Huang, J., Nualart, D., and Tindel, S. Stochastic heat equations with general multiplicative Gaussian noises: Hölder continuity and intermittency. Electron. J. Probab., 20, no. 55, 50 (2015). MR3354615.

$\mathrm{Hu}, \mathrm{Y}$. and Nualart, D. Renormalized self-intersection local time for fractional Brownian motion. Ann. Probab., 33 (3), 948-983 (2005). MR2135309.

$\mathrm{Hu}, \mathrm{Y}$. and Nualart, D. Stochastic heat equation driven by fractional noise and local time. Probab. Theory Related Fields, 143 (1-2), 285-328 (2009). MR2449130.

Huang, J., Lê, K., and Nualart, D. Large time asymptotics for the parabolic Anderson model driven by space and time correlated noise. Stoch. Partial Differ. Equ. Anal. Comput., 5 (4), 614-651 (2017a). MR3736656.

Huang, J., Lê, K., and Nualart, D. Large time asymptotics for the parabolic Anderson model driven by spatially correlated noise. Ann. Inst. Henri Poincaré Probab. Stat., 53 (3), 1305-1340 (2017b). MR3689969.

Huang, J., Nualart, D., and Viitasaari, L. A central limit theorem for the stochastic heat equation. Stochastic Process. Appl., 130 (12), 7170-7184 (2020a). MR4167203.

Huang, J., Nualart, D., Viitasaari, L., and Zheng, G. Gaussian fluctuations for the stochastic heat equation with colored noise. Stoch. Partial Differ. Equ. Anal. Comput., 8 (2), 402-421 (2020b). MR4098872.

Khoshnevisan, D., Nualart, D., and Pu, F. Spatial stationarity, ergodicity and clt for parabolic anderson model with delta initial condition in dimension $d \geq 1$. ArXiv Mathematics e-prints (2020). arXiv: 2007.01987.

Mémin, J., Mishura, Y., and Valkeila, E. Inequalities for the moments of Wiener integrals with respect to a fractional Brownian motion. Statist. Probab. Lett., 51 (2), 197-206 (2001). MR1822771.

Nourdin, I. and Peccati, G. Normal approximations with Malliavin calculus. From Stein's method to universality, volume 192 of Cambridge Tracts in Mathematics. Cambridge University Press, Cambridge (2012). ISBN 978-1-107-01777-1. MR2962301.

Nualart, D. The Malliavin calculus and related topics. Probability and its Applications (New York). Springer-Verlag, Berlin, second edition (2006). ISBN 978-3-540-28328-7; 3-540-28328-5. MR2200233.

Nualart, D. and Peccati, G. Central limit theorems for sequences of multiple stochastic integrals. Ann. Probab., 33 (1), 177-193 (2005). MR2118863.

Nualart, D. and Zheng, G. Averaging Gaussian functionals. Electron. J. Probab., 25, Paper No. 48, 54 (2020a). MR4092767.

Nualart, D. and Zheng, G. Spatial ergodicity of stochastic wave equations in dimensions 1, 2 and 3. Electron. Commun. Probab., 25, Paper No. 80, 11 (2020b). MR4187721.

Nualart, D. and Zheng, G. Spatial ergodicity of stochastic wave equations in dimensions 1, 2 and 3. Electron. Commun. Probab., 25, Paper No. 80, 11 (2020c). MR4187721.

Peccati, G. and Tudor, C. A. Gaussian limits for vector-valued multiple stochastic integrals. In Séminaire de Probabilités XXXVIII, volume 1857 of Lecture Notes 
in Math., pp. 247-262. Springer, Berlin (2005). MR2126978.

$\mathrm{Pu}, \mathrm{F}$. Gaussian fluctuation for spatial average of parabolic Anderson model with Neumann/Dirichlet boundary conditions. ArXiv Mathematics e-prints (2020). arXiv: 2008.08267.

Song, J., Song, X., and Xu, F. Fractional stochastic wave equation driven by a Gaussian noise rough in space. Bernoulli, 26 (4), 2699-2726 (2020). MR4140526. 